\title{
Zur spätpleistozänen Vegetationsentwicklung der Banquette von Barraux (Grésivaudan, französische Nordalpen)
}

\author{
Peter Peschke*) \\ Mit Beiträgen zur Reliefgenese (Christian Hannss) und Klimarekonstruktion (STEFAn Klotz)
}

Peschke, P. (2000), mit Beiträgen von C. Hansss (Reliefgenese) und S. Kıотz (Klimarekonstruktion): Zur spätpleistozänen Vegetationsentwicklung der Banquette von Barraux (Grésivaudan, französische Nordalpen). Eiszeitalter und Gegenwart, 50: 1-24; Hannover 2000.

Keywords: Upper Pleistocene, last interglacial, earliest Weichselian, pollenanalysis, lithostratigraphy, banquette, Pleistocene sedimentation cycle, climate reconstruction, Grésivaudan, northern French Alps, Isère.

Kurzfassung: In dem in den französischen Nordalpen gelegenen und von der mittleren Isère durchflossenen Grésivaudan wurde pollenanalytisch „Unterhalb La Flachère" in rund $330 \mathrm{~m}$ Höhe eine durchlaufende, $10 \mathrm{~m}$ mächtige letztinterglazial- bis frühestweichselzeitliche fossilführende Feinsedimentsequenz nachgewiesen. Sie wird vom Eem (OIS $5 \mathrm{e}$ ), dem St. Germain I und II (OIS 5c, 5a) sowie dem ältesten frühestweichselzeitlichen Kiefern-Fichten-Interstadial (OIS 5a-4) gebildet Erstmals fand sich hier der rein pollenanalytische Beweis, dass auch inneralpin in der collinen Stufe in allen letztinterglazialzeitlichen Warmphasen thermophile Baumarten vertreten sein können. Die Feinsedimentabfolge von Unterhalb La Flachère ist Bestandteil eines kompletten pleistozänen Sedimentationszyklus. Er reicht von der Saale- (= OIS 6) bis zur Weichselmoräne (OIS 2,4?) und verweist auf eine besonders mächtige Talverschüttung im Frühestweichsel (Übergangsbereich OIS 5a-4). Mittels der Methode der modernen Analoge, bei der die Klimarekonstruktion zu fossilen Pollenspektren durch den Vergleich mit rezenten Oberflächenspektren und den dazugehörenden klimatischen Daten erfolgt, wurden die pollenanalytischen $\mathrm{Er}$ gebnisse in Paläoklimawerte überführt.

\section{[Late Pleistocene vegetational development of the Banquette of Barraux (Grésivaudan, French northern Alps)]}

Abstract: In the Grésivaudan situated in the French northern Alps and crossed by the middle course of the river Isère, a complete $10 \mathrm{~m}$ high sequence of fossiliferous fine sediments at "Below La Flachère" (about 330 $\mathrm{m}$ a. s. 1.) was dated by pollen analysis ranging from the last interglacial to the very early Weichselian period. It

* Anschriften der Verfasser: Prof. Dr. Chr. Hannss, Geographisches Institut, Universität Tübingen, Hölderlinstr. 12, D-72074 Tübingen; Dr. St. KLotz, Institut für Geologie, Universität Tübingen, Sigwartstr. 10, D-72076 Tübingen; Dr. P. Peschke, Institut für Botanik, Uni Hohenheim (210), D-70593 Stuttgart covers the Eemian (OIS 5e), St. Germain I and II (OIS $5 c, 5 a)$ and the oldest pine-spruce interstadial (OIS $5 \mathrm{a}-4)$ of the very early Weichselian period. For the first time it was possible to prove by pollen analysis only that thermophilous tree species can be represented in all warm phases of the last interglacial even within the Alps in the colline stage. The sequence of the fine sediments of "Below La Flachère" is part of a complete Pleistocene sedimentation cycle that reaches from the Saale (= OIS 6) to the Weichsel moraine (OIS 4?, 2). It points to a huge valley accumulation in the very early Weichselian period (OIS 5a-4). With the help of the modern analogue method which reconstructs the climate on the basis of fossil pollen spectra via the comparison to recent surface samples, the pollen analytic results were transformed in paleoclimatic clates.

\section{Der geomorphologische und litho- stratigraphische Rahmen (Christian Hannss)}

Das pollenanalytisch bearbeitete Vorkommen von Unterhalb La Flachèrel liegt rund $37 \mathrm{~km} \mathrm{NE}$ von Grenoble auf der Westseite des Hoch-Grésivaudan im Bereich der zentralen Banquette von Barraux in rund $330 \mathrm{~m}$ Höhe (Abb. 1). Bei den Banquetten handelt es sich um terrassenähnliche Reliefelemente. Im Unterschied zu echten fluviatilen Terrassen weisen sie eine gewellte Oberfläche auf, da sie per definitionem eisüberfahren sind. Sie bestehen häufig aus Ablagerungen mehr oder weniger kompletter pleistozäner Sedimentationszyklen (Gremmen \& Hannss 1994: 31, 32). Das heißt, dass über einer Liegendmoräne die Füllung eines verlandenden Eisrückzugsees akkumuliert wurde, die von interglazialzeitlichen Sedimenten meist in der Form von Moor- beziehungsweise Gyttja-Ablagerungen überdeckt wird, die reich an organischer Substanz sind. Diese wiederum werden häufig noch diskordant von Vorstoßschottern und einer Hangendmoräne überlagert. Im Bereich der Banquette von Barraux spiegelt die Sequenz von La Gache (Hannss 1982: 60) diese systematische Abfolge lithostratigraphisch größten-

1) $50^{\circ} 441$ Nord, $43^{\circ} 050$ östlich Paris nach Carte de France 1:25 000, Montmélian $\mathrm{N}^{\circ}$ 7-8. 


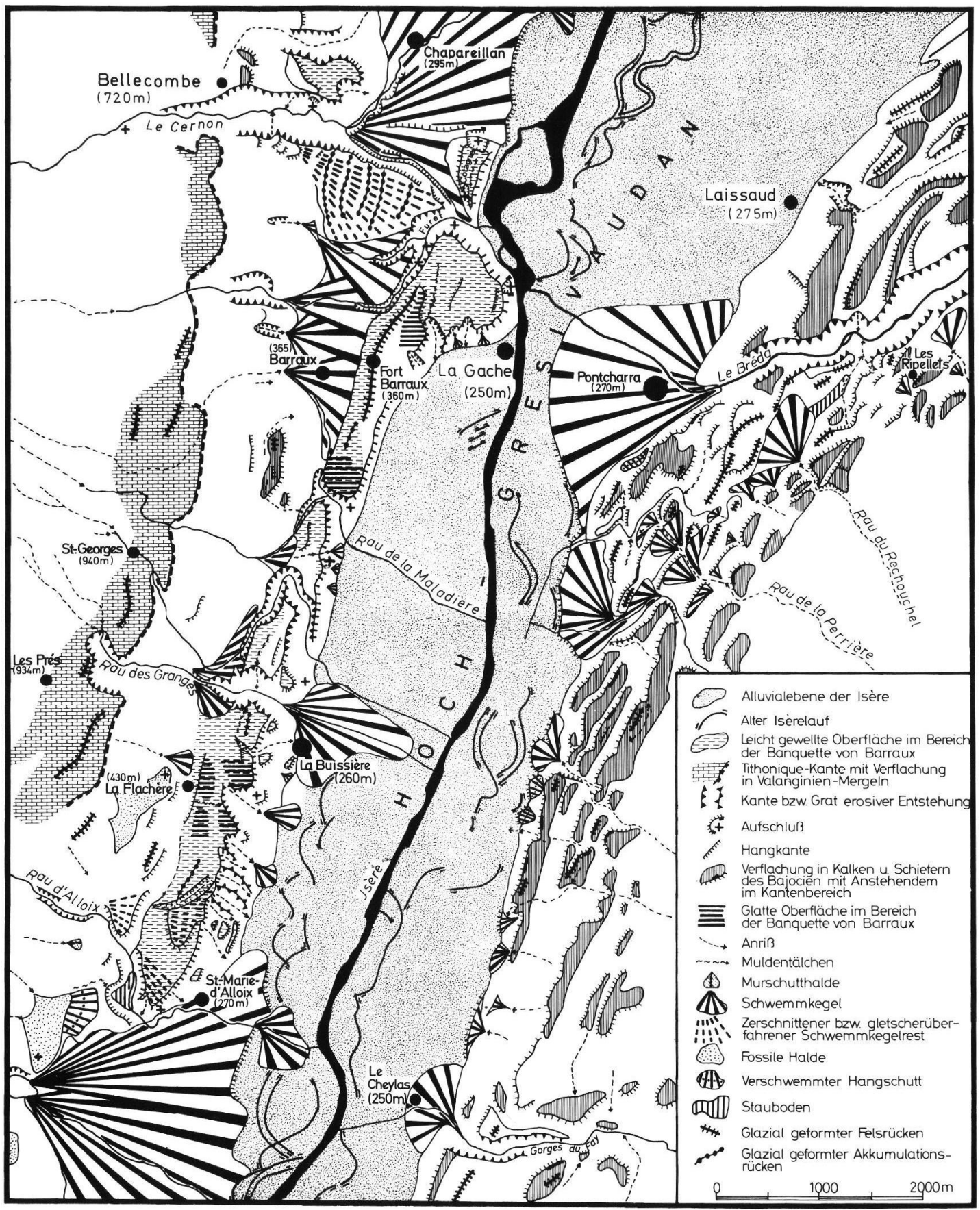

Abb. 1: Geomorphologische Übersichtskarte des Hochgrésivaudan. - Kartographie: G. KocH

Fig. 1: Map of the main geomorphological features from the High Grésivaudan. - Cartography: G. KocH.

teils wider. Aber auch die anderen Aufschlüsse dieser Banquette fügen sich in den pleistozänen Sedimentationszyklus ein.

Die Basis der Quartärablagerungen der Banquette von Barraux (Abb. 2) bildet eine kalkreiche
Saalegrundmoräne (= OIS 6)2. Überdeckt wird sie von teilweise scharfkantigen geschiebeführenden, teilweise aber auch von Grobbestandteilen freien und zumindest im Furet-Anriss pollenleeren $(\mathrm{CH}$. REYNAUD nach mündlicher Auskunft von 
G. MonjuvenT), tonig-siltigen glaziolakustren Akkumulationen, die der auslaufenden Saaleeiszeit zuzurechnen sind.

Zwischen annähernd $310 \mathrm{~m}$ und $336 \mathrm{~m}$ folgen in unterschiedlicher facieller Ausprägung die Ablagerungen des letzten Interglazials, zu denen zwischen $325 \mathrm{~m}$ und $336 \mathrm{~m}$ auch die neu pollenanalytisch bearbeitete, teilweise Ostracoden führende (mündliche Mitt. CH. Hemleben) limnische bis Bruchwaldtorf-Sequenz von Unterhalb La Flachère gehört (Abb. 3). Sie lagert wahrscheinlich transgressiv der Saalemoräne auf. Das letztinterglazialzeitliche Niveau von Unterhalb La Flachère wird in großer Mächtigkeit noch von sandigen Akkumulationen der auslaufenden Saaleeiszeit unterlagert.

Auf letztinterglazialzeitliche Ablagerungen stößt man im Grésivaudan in gleicher Facies wie Unterhalb La Flachère auch noch auf seiner Ostseite, und zwar mit $335 \mathrm{~m}$ bis $340 \mathrm{~m}$ in nahezu der gleichen Höhe wie die letztinterglazialzeitliche Gesamtserie rechts der Isère (Hannss 1982: 35 ff). Oberhalb von Brignoud konnte GrEmmen (1982: 69, 14) Akkumulationen aus seinem mit dem St. Germain I korrelierten auslaufenden Tremblay-Interstadial nachweisen bzw. sein Bourget II-Stadial, das er dem Melisey II von WoILlARD (1978: 3) gleichgesetzt hat.

Im nördlichen Bereich der Banquette von Barraux finden sich die letztinterglazialzeitlichen $\mathrm{Ab}-$ lagerungen in der großen Kiesgrube von La Gache oberhalb der endsaalezeitlichen Deltaschüttung zwischen $300 \mathrm{~m}$ und $310 \mathrm{~m}$ in den hier in wechselnder Mächtigkeit verstärkt auftretenden sandig bis tonig-siltigen Linsen (Havnss 1982: 60). Belege dafür sind jedoch nur drei in $310 \mathrm{~m}$ Höhe von M. Coursange geborgene Fichtenhölzer (Bestimmung F. Schweingruber), Holzkohle in $305 \mathrm{~m}$ Höhe sowie nach Bourdier (1962: 24, fig. 24) zwischen $300 \mathrm{~m}$ und $310 \mathrm{~m}$ beobachtete Bruchstücke von Mollusken beziehungsweise Spuren anderer organischer Reste.

Ebenfalls im nördlichen Teil der Banquette von Barraux fanden Depape \& Bourdier (1952a: 1532;

\footnotetext{
2) OIS = Isotopenstadium. In Anlehnung an SCHLÜCHTER (1976: Tafel I) wurde in diesem Artikel zur Bezeichnung der verschiedenen pleistozänen Zeitepochen die mitteleuropäische Nomenklatur verwendet. Dies geschah unter anderem deshalb, weil seit längerer Zeit einige Interglazialbezeichnungen von dort auch für den alpinen Raum verwendet werden und zudem das Riss derzeit chronostratigraphisch nicht näher fassbar ist.
}

1952b: 81) im Furet-Anriss in wahrscheinlich auch hier Schottern inkorporierten sandig bis siltigen Tonen zwischen $300 \mathrm{~m}$ und $310 \mathrm{~m}$ eine interglazialzeitliche Blätterflora mit Rbododendron ponticum, Buxus sempervirens, Tilia, Acer, Carpinus betulus, Alnus incana, A. glutinosa, A. viridis und Abies pectinata. In diesem Anriss hatte leider ohne Angaben zur Höhe und Lithostratigraphie bereits 1851 Gras (S. 198) 30-40 cm mächtige Schieferkohlen beschrieben, die wegen der in ihnen enthaltenen Eichenhölzer eigentlich auch nur in das letzte Interglazial gehören können (zur Lithostratigraphie siehe Hanvss 1982: 43).

Ganz im Nordteil der Banquette von Barraux wurde von Bottema und Koster (in Hannss et al. 1976: $500,503-505)$ in $312 \mathrm{~m}$ in Cotagnier-Süd pollenanalytisch eine kurze letztinterglazialzeitliche Sequenz mit hohen Picea-, Pinus- und Abies-Werten nachgewiesen und zwar in einer Sanden bzw. Schottern eingelagerten Ton- bis Schieferkohlenlinse. Diese palynologischen Ergebnisse werden gestützt durch 50 in ihnen geborgene und von F. Schweingruber bestimmte Fichtenhölzer (Hannss 1982: Tab. 6). Die Sequenz wurde von Gremmen 1982 (S. 64-65, diagr. 9) neu bearbeitet und unter Vorbehalt mit seinem Rau des Combes-Interglazial, also dem auslaufenden Eem, korreliert. Diese Einschätzung fand seine Bestätigung durch die von Peschke Unterhalb La Flachère nachgewiesene Pollenflora (= Pollenzone $\mathrm{b}$ ) des oberen Eems (Abb. 7).

An der Nordgrenze der Banquette von Barraux fällt das letztinterglazialzeitliche Niveau höchstwahrscheinlich mit den zwischen $292 \mathrm{~m}$ und $310 \mathrm{~m}$ in der alten Tongrube von Chapareillan beobachtbaren Baumstammlagen zusammen, die Schottern eingelagert sind (Hannss 1982: 56). Bei den bestimmbaren Holzresten handelt es sich nach W. SchOCH um Abies. Dies stünde in Übereinstimmung mit dem relativ hohen Pollenanteil der Tanne, der in Cotagnier-Süd im 312 m-Niveau in der dort freigelegten Ton-Schieferkohlenlinse festgestellt worden ist.

Soweit das letztinterglazialzeitliche Niveau im Bereich der Banquette von Barraux nicht selbst schon in fluviatilen Schottern entwickelt ist, werden die in limnischer Facies vorliegenden Akkumulationen der letzten Warmzeit von frühestweichselzeitlichen Schottern überlagert. Diese gliedert Unterhalb La Flachère in rund $348 \mathrm{~m}$ Höhe eine Bank siltig-toniger Ablagerungen. In ihnen wurde ein von F. SCHwEIngruber bestimmtes Fichtenstammholz geborgen. Die Pollenanaly- 


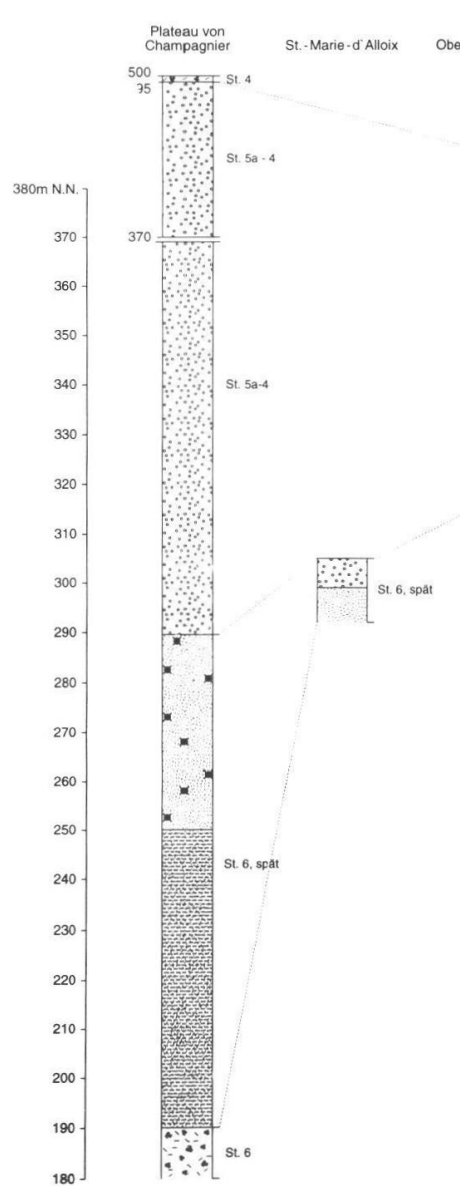

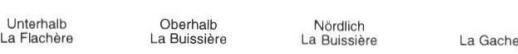

Fure!

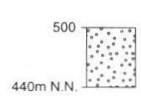

Alte Tongrube
Chaparallilan

Les Marches
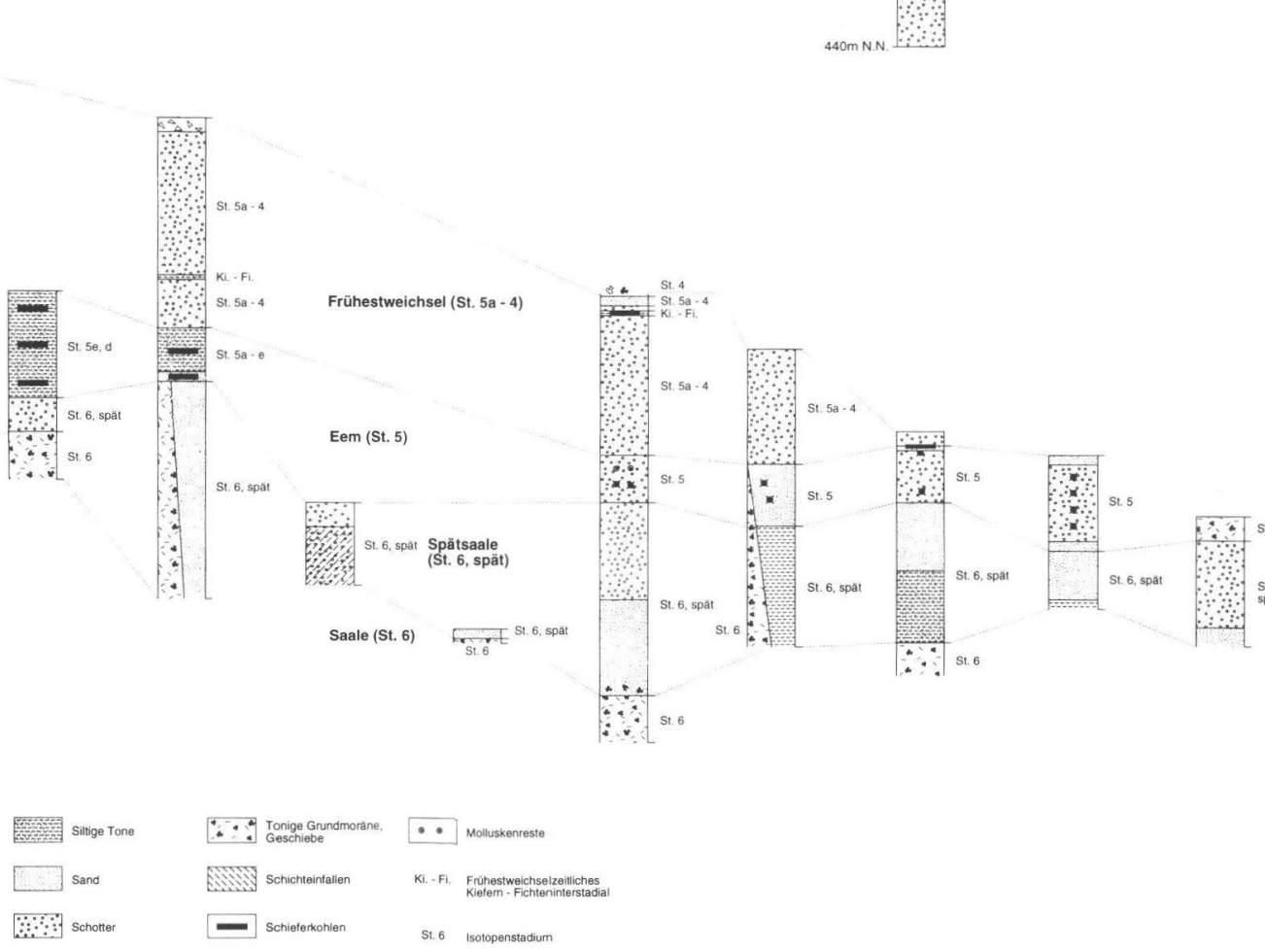

Saale (St. 6)
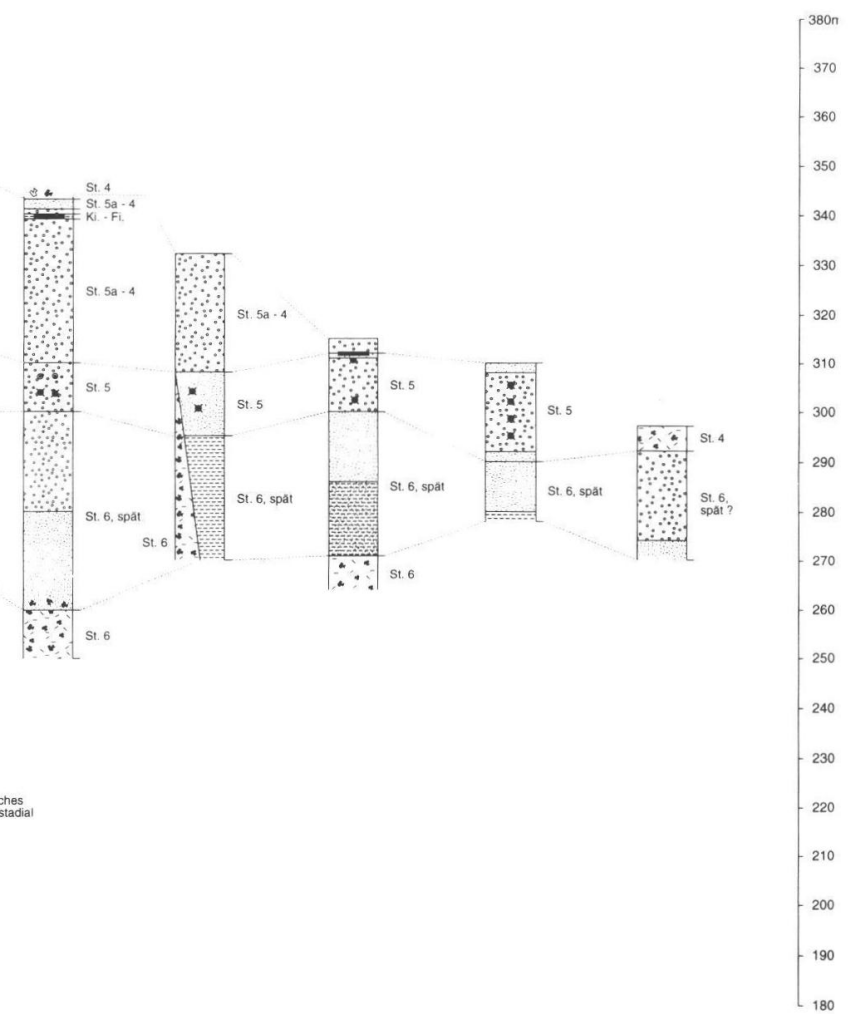

Abb. 2: Quartärstratigraphische Übersicht des Hochgrésivaudans und des Beckens von Grenoble; kotenmäßßige Rekonstruktion von Profilabschnitten. - Kartographie: R. SZYDLAK

Fig. 2: Main structures of the Quaternary stratigraphy of the High Grésivaudan and of the basin of Grenoble; correlation of selected sections by its elevation a. s. 1. Cartography: R. SZYDL.AK. 
(5)

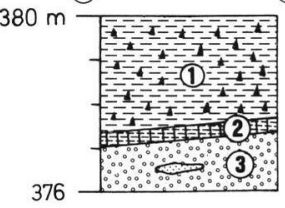

358,5

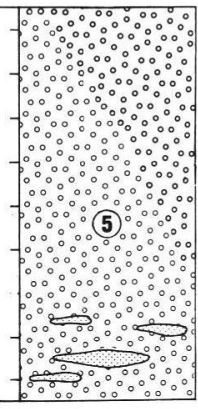

349,5
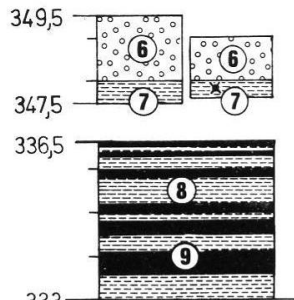

(11)
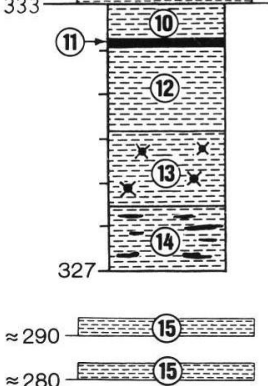

(N) Abb. 3: Lithostratigraphisches Sammelprofil der Quartärablagerungen von Unterhalb La Flachère. - Kartographie: G. KосH

1 = Weichselstadialzeitlicher, lokaler Kalkhangschutt mit fein- bis mittelkiesigen, kantigen Einzelkomponenten.

2 = Lehmig-tonige Zwischenlage lokaler Provenienz mit einem Kalkanteil von $48 \%$.

3 = Ungeschichtete Schotter fast ohne Kalkgerölle.

5 = Ungeschichtete Grobschotter mit pollenfreien Feinsedimentlinsen.

$6=$ Konglomeratisierte Schotterlage.

7 = Ungeschichtete, fossilführende frühestweichselzeitliche Tone mit einem von F. Schweingruber bestimmten Picea-Holz. Pollenanalyse siehe Abb. 4.

8 = Letztinterglazialzeitliche bis frühestweichselzeitliche, ungeschichtete, graublaue Tone.

9 = Letztinterglazialzeitliche bis frühestweichselzeitliche Schieferkohlen mit Holzresten.

10 = Letztinterglazialzeitliche, fossilführende, graublaue Tone.

11 = Letztinterglazialzeitliche, kompakte Schieferkohlen.

12 = Letztinterglazialzeitliche, sterile graublaue, leicht siltige Tone.

13 = Letztinterglazialzeitliche, fossilführende graue, z. T. siltige Tone.

14 = Letztinterglazialzeitliche, fossilführende graue Tone mit Schieferkohlenlagen.

15 = Endsaalezeitliche Sande.

Pollenanalyse der Schichtglieder 8 bis 14 siehe Abbildungen 5 bis 7 .

Fig. 3: Compiled lithostratigraphic section of the Quaternary deposits from „Below La Flachère“. - Cartography : G. KocH.

$1=$ Weichselian local calcareous talus debris consisting of angular fine- and medium pebbles

$2=$ Loam-clay interlayer of local origin with lime content of $48 \%$

3 = Non-stratified gravel almost without calcareous pebbles

$5=$ Non-stratified coarse gravel with lenses of fines free of pollen

$6=$ Conglomerate

$7=$ Earliest Weichselian non-stratified fossiliferous clay with wood of Picea (determined by F. Schweingruber). For pollen analysis see Fig. 4.

$8=$ Last Interglacial to earliest Weichselian non-stratified grey-blue clay

$9=$ Last Interglacial to earliest Weichselian slate coal with remnants of wood

10 = Last Interglacial blue-grey fossiliferous clay

$11=$ Last Interglacial compact slate coal

12 = Last Interglacial sterile blue-grey and slightly silty clay

13 = Last Interglacial fossiliferous blue-grey clay, partly silty

14 = Last Interglacial fossiliferous grey clay with beds of slate coal

$15=$ End Saalian sand.

For pollen analysis of units 8 to 14 see Figs. 5 to 7.

Tab. 1: Pollenflora der fossilführenden Feinablagerung des 340-m-Niveaus der Kiesgrube von La Gache, Nordteil der Banquette von Barraux. Nach schriftl. Mitteilung (27.10.1980) von J.-L. De Beauliet.

Tab. 1: Pollenanalytical results of the fossiliferous fine sediments at the $341 \mathrm{~m}$ level from the gravel pit of La Gache, northern part of the Banquette of Barraux. After written communication (27. 10. 1980) from J.-L. De BeAluleu.

\begin{tabular}{|c|c|c|c|c|c|c|c|c|c|}
\hline $\begin{array}{l}\text { Proben- } \\
\text { nummern }\end{array}$ & $\begin{array}{c}\text { Betula } \\
\%\end{array}$ & $\begin{array}{c}\text { Pinus } \\
\% \\
\end{array}$ & $\begin{array}{c}\text { Picea } \\
\% \\
\end{array}$ & $\begin{array}{c}\text { Abies } \\
\% \\
\end{array}$ & $\begin{array}{c}\text { Alnus } \\
\%\end{array}$ & $\mathrm{AP}$ & $\begin{array}{c}\text { Gramineae } \\
\% \\
\end{array}$ & $\begin{array}{c}\text { Cyperaceae } \\
\% \\
\end{array}$ & $\begin{array}{c}\text { Pollen- } \\
\text { summe } \\
=100 \%\end{array}$ \\
\hline 1543 & 7 & 16 & 21 & - & 8 & 54 & 12 & 9 & 350 \\
\hline 1542 & - & 17 & 45 & 1 & 3 & 68 & 15 & 15 & 240 \\
\hline 1561 & - & 35 & 21 & 3 & 7 & 65 & 18 & 8 & 145 \\
\hline $\begin{array}{l}\text { unten } \\
1566\end{array}$ & 1 & 14 & 36 & 3 & - & 74 & 5 & 14 & 260 \\
\hline oben & & & & & & & & & \\
\hline 1539 & 2 & 17 & 19 & 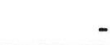 & 8 & 46 & 26 & 14 & 235 \\
\hline
\end{tabular}




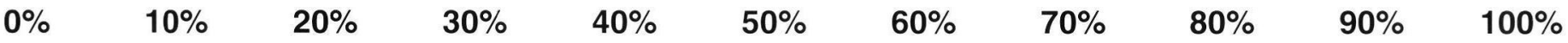

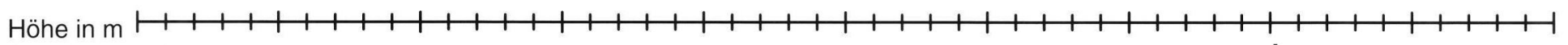
über NN

348,3

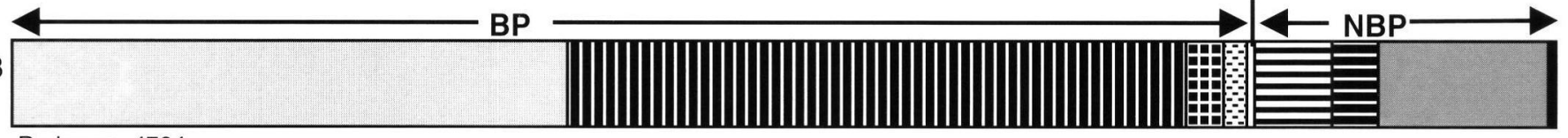

Probennr. 4764

348,1

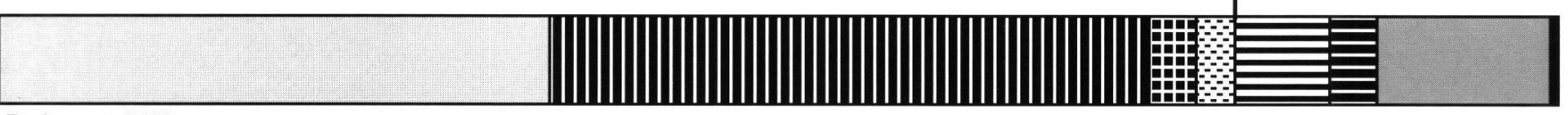

Probennr. 4760

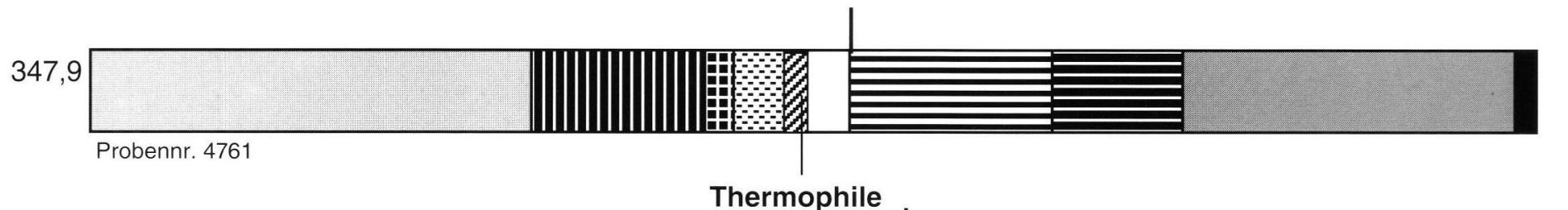

Thermophile

347,7
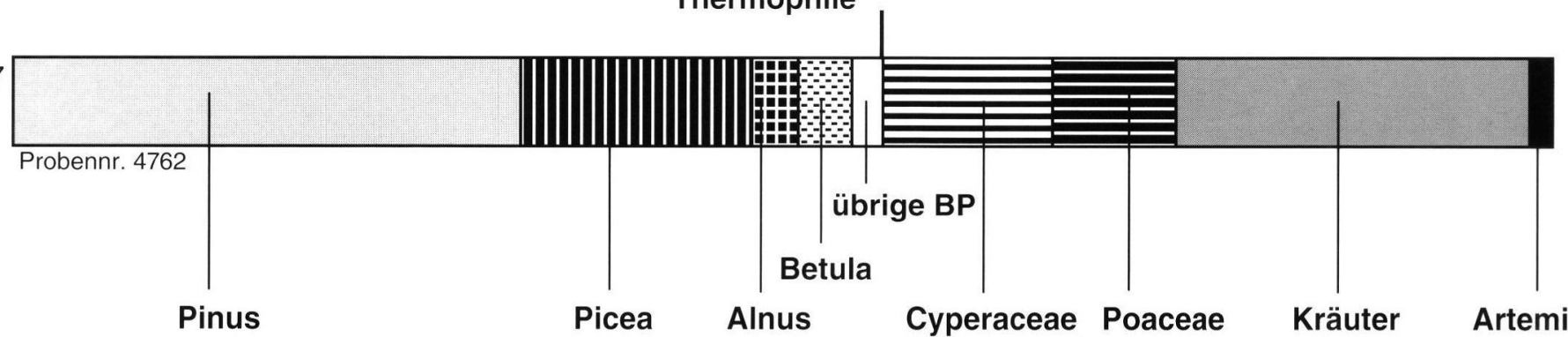

474

Abb. 4: Pollendiagramm der frühestweichselzeitlichen Ton-Siltbank zwischen 347,5 m und 348,5 m Unterhalb La Flachère (PeschkE). Grundsumme $=$ BP + NBP inklusive Gramineae und Poaceae. - Kartographie: F. VETTER.

Fig. 4: Pollen diagram of the earliest Weichselian silt-clay bed between $m$ 347,5-348,5 from „Below La Flachèr” (PeschKE). Total sum = AP + NAP including Gramineae and Poaceae. - Cartography: F. VETTER. 
se von Peschke (Abb. 4) hat ergeben, dass diese Feinakkumulationen zu einem der frühestweichselzeitlichen Kiefern-Fichten-Interstadiale gehören. Ein derartiges Interstadial (Tab. 1) wurde in ähnlicher Höhe in $340 \mathrm{~m}$ pollenanalytisch bereits früher in der Kiesgrube von La Gache durch DE Beaulieu (Hannss 1983: 24) in einem Ton- bis Schieferkohlenband nachgewiesen, das frühestweichselzeitlichen Schottern zwischengeschaltet ist.

Dass es sich bei den in $340 \mathrm{~m}$ entwickelten fossilführenden Feinablagerungen von La Gache nicht um eine mittelweichselzeitliche Bildung handeln kann, zeigt vor allem die starke Verbreitung der Fichte, die im Mittelweichsel (OIS 3) in den Alpen so gut wie nicht mehr vorkommt (BRun, Hannss, 1998: 12). Dies steht in Übereinstimmung mit der für die fossilführende 340-m-Schicht von A. MavGINI erzielten U-Th-Datierung von $98000 \mathrm{v}$. h. sowie prinzipiell auch mit einer ${ }^{14} \mathrm{C}-\mathrm{D}$ atierung von $59900 \pm 850 \mathrm{BP}$, die aber nur als Radiokarbon- mindestalter gedeutet werden kann (Tab. 2). Letztlich haben alle im Bereich der Banquette von Barraux angestellten neueren quartärwissenschaftlichen Studien im Grundsatz die bereits von BOurdier (1961/62) auf der Grundlage noch vergleichsweise einfacher Untersuchungsmethoden aufgestellte Hypothese bestätigt, dass die Banquette-Ablagerungen im Grésivaudan wie im Becken von Grenoble im Verlauf eines durchlaufenden pleistozänen Sedimentationszyklus akkumuliert worden sind, der vom vorletzten Glazial (OIS 6) bis zum letzten, dem weichselzeitlichen Glazial des Isotopenstadiums 4 oder 2 reicht (Abb. 2). Aufgrund der jüngst im Vercors (BEAuLIEU, J.-L. DE et al. 1994: 128, 137), im Val du Bourget (unveröffentlicht), in Niedersachsen (UrBAN 1996: 494) und in Sachsen (EISSMann 1997: 46; KRBETSCHEK \& STOLz 1994: 294) erzielten Resultate ist dabei davon auszugehen, dass die kalkreiche Liegendmoräne der Banquette von Barraux in das OIS Stadium $6 \mathrm{zu}$ stellen ist und mit dem maxi-

Tab. 2: Radiokarbondatierungen jungpleistozäner Ablagerungen der Banquette von Barraux.

Tab. 2: Radiocarbon dates of late Pleistocene deposits of the Banquette of Barraux.

\begin{tabular}{|c|c|c|c|}
\hline Lokalität & $\begin{array}{c}\text { Konventionelles }{ }^{14} \mathrm{C}- \\
\text { Alter B.P. mit } \\
\text { Labornummer in } \\
\text { Klammer }{ }^{1)}\end{array}$ & $\begin{array}{c}\text { Vermutliches } \\
\text { klimastratigraphisches } \\
\text { Alter mit OIS }\end{array}$ & $\begin{array}{l}\text { Datiertes Material } \\
\text { und Labor }\end{array}$ \\
\hline $\begin{array}{l}\text { Unterhalb La Flachère, } \\
333-336 \mathrm{~m}\end{array}$ & $\begin{array}{l}<21100(\mathrm{C}-588)^{3)} \\
<35000^{4)} \\
<45000(\mathrm{Ly}-900)^{5)} \\
<56400(\mathrm{Gr} N-6652)^{6)} \\
65300-1400+1700 \\
(\mathrm{Gr} N-7222)^{6)}\end{array}$ & $\begin{array}{l}\text { Eem, St. Germain I und } \\
\text { II } \\
(5 \mathrm{a}, \mathrm{c}, \mathrm{e})\end{array}$ & $\begin{array}{l}\text { Holz (?) } \\
\text { Holz (Ly) } \\
\text { Holz (Ly) } \\
\text { Holz (Gr) } \\
\text { Abies (Gr) }\end{array}$ \\
\hline $\begin{array}{l}\text { Oberhalb Brignoud, } \\
330 \mathrm{~m}\end{array}$ & $\begin{array}{l}<500000^{7)} \\
<\underline{60000 \pm 600^{8)}}\end{array}$ & $\begin{array}{l}\text { St. Germain II } \\
(5 \mathrm{a})\end{array}$ & $\begin{array}{l}\text { Tonige } \\
\text { Schieferkohle (H) } \\
\text { Tonige } \\
\text { Schieferkohle (Gr) }\end{array}$ \\
\hline $\begin{array}{l}\text { Kiesgrube La Gache, } \\
340 \mathrm{~m}\end{array}$ & $\begin{array}{l}<50000^{6)} \\
\underline{59900 \pm 850^{7)}}\end{array}$ & $\begin{array}{l}\text { Frühestweichsel- } \\
\text { Interstadial } \\
\text { (Übergang 5a-4) }\end{array}$ & $\begin{array}{l}\text { Tonige } \\
\text { Schieferkohle (Gr) } \\
\text { Tonige } \\
\text { Schieferkohle (Gr) }\end{array}$ \\
\hline $\begin{array}{l}\text { Cotagnier-Sud, } \\
310 \mathrm{~m}\end{array}$ & $<{\underline{48900^{6}}}^{6}$ & $\begin{array}{l}\text { St. Germain II } \\
\text { (5a) }\end{array}$ & Picea (Gr) \\
\hline $\begin{array}{l}\text { Alte Tongrube von } \\
\text { Chapareillan, } \\
295-310 \mathrm{~m}\end{array}$ & $\begin{array}{l}40800-2500+ \\
3800^{9)}\end{array}$ & $\begin{array}{l}\text { Letztes Interglazial } \\
\text { (5) }\end{array}$ & $\begin{array}{l}\text { Zerfallenes Holz } \\
(\mathrm{H})\end{array}$ \\
\hline
\end{tabular}

1) Bei den unterstrichenen Daten handelt es sich um Anreicherungsdatierungen.

2) $\mathrm{Ly}=$ Lyon, $\mathrm{Gr}=$ Groningen, $\mathrm{H}=$ Heidelberg.

5) Radiocarbon (1976: 66).

3) Radiocarbon (1955: 86).

4) Monjuvent (1969: 550). Labornummer unbekannt.

6) HANnSS et al. (1976: 506).

7) Hannss (1983: 21).

8) Hannss (1982: Tab. 5).

9) Hannss (1974: 553). 
malen saalezeitlichen Vorstoß, dem DrentheStadium sensu LiedtKe (1981, Abb. 7) zusammenfallen dürfte.

Die Konzeption, alle Saale-Stadien in das OIS 6 zu stellen, erfolgte vor dem Hintergrund, dass Uriban (1996: 494) in Schöningen, DE BEAulieu et al. (1994: 137, 141) im Vercors und ReIlle et al. (1998: 1109) im Massif Central mit hoher Wahrscheinlichkeit belegen konnten, dass zwischen dem Holstein- und dem Eem-Interglazial noch zwei weitere, in das OIS 9 und das OIS $7 \mathrm{zu}$ stellende Warmzeiten existiert haben.

Die Hypothese eines zwischen dem St. Germain I und dem St. Germain II (Gremmen 1982: fig. 12, 14) stattgefundenen gewichtigen Gletschervorstoßes, wie er für das Grésivaudan postuliert worden ist (Hannss 1982: 106; 1983: 21, 22) und für diesen Zeitraum zudem von Weltev (1982: 168) in den Schweizer Alpen angenommen wurde, muss nicht zuletzt auch wegen der neuen pollenanalytischen Ergebnisse von Unterhalb La Flachère als widerlegt gelten.

\section{Vorbemerkungen zur Pollenanalyse}

Die wissenschaftliche Kernbohrung Unterhalb La Flachère wurde im August 1991 unmittelbar neben dem Aufschluss niedergebracht, aus welchem das Material für das bereits publizierte Pollenprofil von Gremmen (1982) stammt. Da in einer mit der freundlichen Unterstützung von G. MoNJUVENT 1978 niedergebrachten Sondierungsbohrung noch $6,3 \mathrm{~m}$ Feinsedimente und Schieferkohlen unter dem Aufschluss nachgewiesen werden konnten, wurde 1991 die pollenanalytisch bearbeitete Kernbohrung niedergebracht. Die Basis des Aufschlusses und der obere Ansatzpunkt der Bohrung liegen, nur wenige Meter horizontal von einander entfernt, auf gleicher Höhe (Abb. 3, 7). Die Sedimentabfolge von Aufschluss und Bohrung gehören in den gleichen Ablagerungszyklus.

Die Kernbohrung durchteufte Wechsellagerungen von Tonen und Schieferkohlen unterschiedlicher Mächtigkeit und erreichte in $815 \mathrm{~cm}$ Tiefe wahrscheinlich Moränenmaterial, worauf die Bohrung eingestellt wurde (Abb. 2, 3, 7). Die ab $700 \mathrm{~cm}$ erreichten olivgrauen bis ockerfarbenen Tone waren so gut wie pollenfrei. Auch in den humosen Straten dieses Bereiches war die Pollenführung für eine Interpretation zu gering und bestand nur aus wenigen Pinus-Pollenbruchstücken, perisporlosen Farnsporen und vereinzelten dickwandigen Asteraceen-Pollenkörnern.
Eine befriedigende auswertbare Pollenführung setzte bei $690 \mathrm{~cm}$ in den Tonen mit höheren humosen Anteilen („Schieferkohlentonen“) ein.

Die Aufbereitung der Sedimente für die Pollenanalyse wurde nach der im Botanischen Institut der Universität Hohenheim üblichen Methode vorgenommen, wobei als schwere Lösung bei der Schweretrennung anstelle von dem schwierig zu entsorgenden Cadmiumjodid Natriumpolywolframat verwendet wird. Die Analyse des gewonnenen Rückstandes erfolgte in Glyzerin bei meist 400facher Vergrößerung, gelegentlich musste bei schwieriger Diagnose Ölimmersion mit 1000facher Vergrößerung benutzt werden. Nach Möglichkeit wurde eine Auszählsumme von 600 bis 800 Pollenkörnern angestrebt, oft liegt die Auszählsumme noch höher.

Das Pollenprofil wurde in zwei Varianten dargestellt; einmal der herkömmliche Typ (Abb. 6) mit Gesamtdiagramm (Grundsumme BP + NBP = 100\%, exklusive Sporen und Wasserpflanzen-Pk) und einem Baumpollendiagramm (Grundsumme BP ohne Almus $=100 \%$ ).

Eine weitere Diagrammdarstellung (Abb. 5) zeigt das Pollenprofil analog der Berechnungs- und Darstellungsweise (Grundsumme $=\mathrm{BP}+\mathrm{NBP}$ ohne Gramineae und Poaceae) nach Gremmen (1982), um eine bessere Vergleichsmöglichkeit mit den dortigen Ergebnissen zu erreichen. Die folgende Diagrammbeschreibung bezieht sich nur auf die zweite Variante, kann aber auch zur Beurteilung der anderen Darstellungart dienen, weil die Einteilung in Diagrammabschnitte identisch ist. Lediglich die angegebenen Prozentwerte differieren.

\section{Aufbau des Pollendiagramms Typ Gremmen}

In der Mitte der Darstellung (Abb. 5) befindet sich das Gesamtdiagramm mit Aufteilung des BP/NBP-Verhältnisses sowie den Häufigkeiten der Holzarten Pinus und Picea. Der NBP-Anteil ist horizontal schraffiert. Links vom Gesamtdiagramm sind in unterschiedlich breiten Spalten mit Schattenrissen und 5 facher Überhöhung die Gehölze eingetragen, rechts davon die Kräuter. Der Anteil der Süß- und Sauergräser ist in der NBP-Summe nicht enthalten, aber auf der rechten Seite neben der Spalte Pollensumme (= Berechnungssumme) relativ darauf bezogen graphisch dargestellt. Nach der Häufigkeit kennzeichnender Holzarten und ihrer Entwicklung wurde das Pollendiagramm in 6 Diagrammabschnitte (DA) a-f 

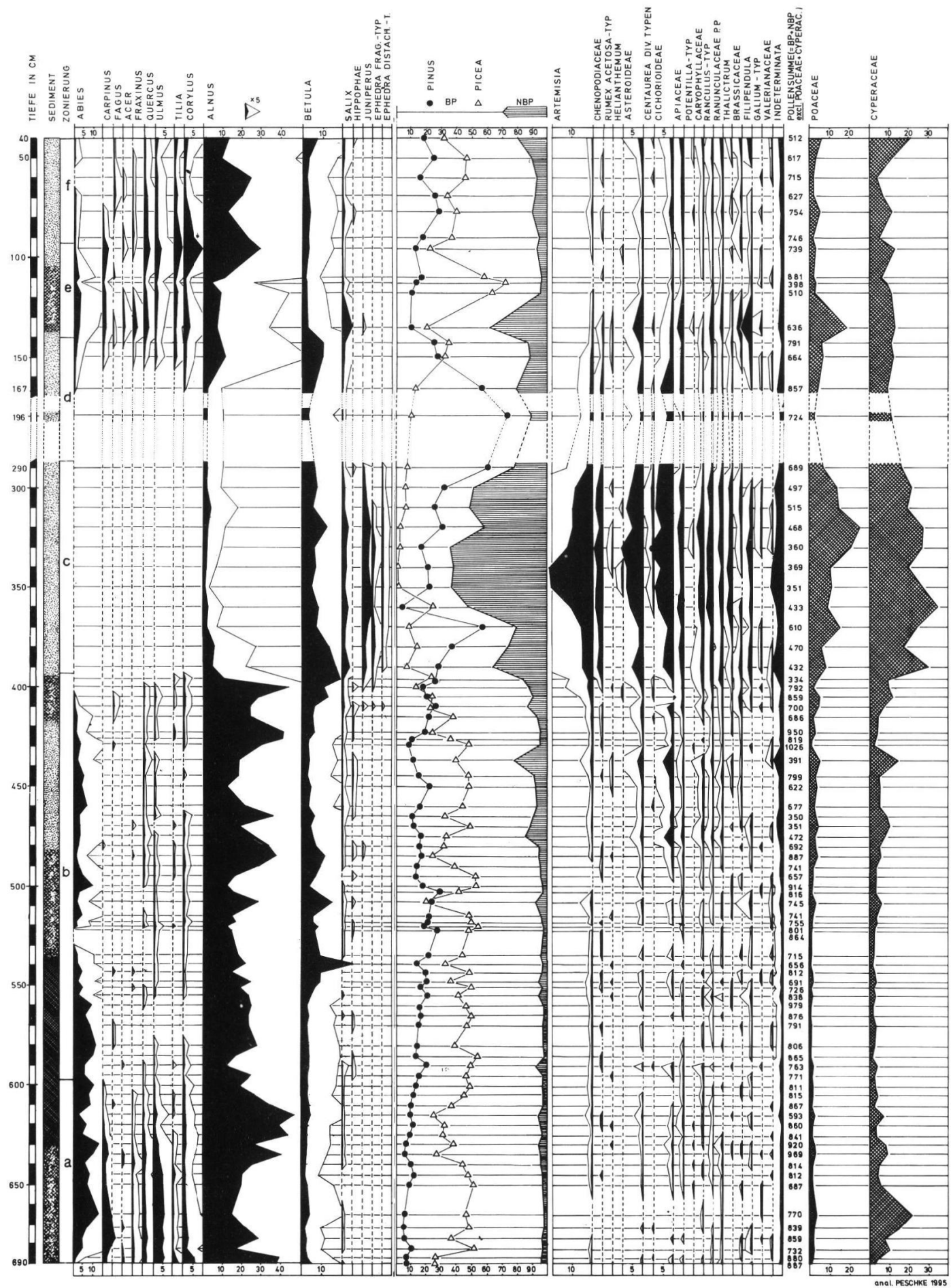

Abb. 5: Pollendiagramm der Kernbohrung von Unterhalb Lá Flachère nach der Darstellungsweise von GREMmEN (1982). Grundsumme = BP + NBP ohne Gramineae und Poaceae. Litostratigraphie: Punkte = Siltige Tone. Schräge Raster $=$ Schieferkohlen. Schräge Raster mit Punkt: Tonige Schieferkohlen. - Kartographie: P. PEschke, G. Koch .

Fig. 5: Pollen diagram of the core "Below La Flachère" after the presentation form of GrEmmEN (1982).

Total sum = AP + NAP without Gramineae and Poaceae. Lithostratigraphy: dots = silty clay; diagonal screen = slate coal, diagonal screen dotted = clayey slate coal. - Cartography: P. Peschke, G. Koch. 
unterteilt. Diese werden im Folgenden von unten nach oben im Einzelnen beschrieben:

\section{Diagrammbeschreibung}

\section{DA a: $\mathbf{9 0 7 , 5}-1000 \mathrm{~cm}$ Profiltiefe 3}

In diesem DA repräsentiert der Pollenniederschlag eine ausgeprägte Waldzeit: Im basalen Spektrum bei $1000 \mathrm{~cm}$ nimmt der BP 96,2\% ein, worin Alnus mit 38,0\% dominiert. EMW-Gehölze, Corylus und Carpinus vertreten neben Almus die Laubwaldflora, der mit 26,5\% Picea und etwas Abies beigemischt sind. Leider sind die Einwanderungsvorgänge dieser Waldvegetationen aufgrund der wahrscheinlich transgressiven lithostratigraphischen Position der fossilführenden Feinsedimente nicht erhalten. Es bleibt völlig offen, was sich vegetationsgeschichtlich seit der $\mathrm{Ab}$ lagerung der Moräne an der Profilbasis und der hier festgestellten Waldzeit ereignet hat.

Der NBP besteht aus dem üblichen Arteninventar geschlossener Wälder ohne irgendwelche charakteristischen Arten oder hohe Pollenwerte. Der Anteil der Sauergräser (Cyperaceae) ist in der unteren Hälfte des DA mehr als doppelt so hoch wie in der oberen, in der Almus mit höheren Werten vertreten ist.

Die Kurvenwerte der thermophilen Gehölze (EMW, Carpinus, Corylus) nehmen zum Ende des DA allmählich ab und setzen zum Teil ganz aus. Offenbar spiegelt sich hier das Ende einer warmzeitlichen Laubwaldflora wider, die ihr Ausbreitungsmaximum schon überschritten hatte. Der relativ hohe Anteil der Fichte beruht auf der Gebirgsrandlage der Lokalität. Vermutlich kam diese Holzart mehr in den oberen Hangpartien und in den Gipfellagen der das Isèretal begleitenden Höhenzüge der Chartreuse und der Belledonne vor. Die Fichte war wahrscheinlich aber auch am Vegetationsaufbau der Tallagen beteiligt, da sie Nassstandorte zu besiedeln vermag und intramontan manchmal einen regelrechten "Fichtenauwald" aufbauen kann. Die hohen Almus-Werte sind Ausdruck einer Bruch- und Auenwaldvegetation in der Talsohle. Außerdem wird die Erle auf ihr zusagenden Standorten in kleinen Seitentälern vorgekommen sein. Die Tanne meidet hingegen Nassstandorte. Dieser Baum wird im Gefolge der Vegetationshöhenstufung eher auf weiter oben gelegenen, absonnigen Hangpartien gewachsen sein, während die thermophilen Laubgehölze

3) $\mathrm{DA}=$ Diagrammabschnitt. Die Tiefenangaben der einzelnen Diagrammabschnitte beziehen sich auf das Gesamtpollenprofil der Abb. 7. hauptsächlich im unteren Teil der nach SE gewandten Hangflanken der Chartreuse vorgekommen sein dürften.

Parallel zum Ausklingen der thermophilen Gehölze wird die Fichte häufiger. Ihr Anteil an der Waldfläche muss zugenommen haben. Hinweise auf offene Flächen im Wald fehlen. Der BP-Anteil am Pollenniederschlag bleibt am DA-Ende unverändert; ausgesprochene Heliophyten im Kräuterpollen kommen nicht vor. Dieser Wechsel der Holzarten, das heißt das Verschwinden der Thermophilgehölze, zeichnet sich im Sediment nicht ab. Die Grenze DA a/b liegt in ungestörten, tonigen, dichtgelagerten, Schieferkohlen.

\section{DA b: $\mathbf{7 0 0}$ - 907,5 cm Profiltiefe}

Die sehr heterogenen Sedimente dieser rund $2 \mathrm{~m}$ langen Bohrstrecke sind tonige Schieferkohlen, humose Tone und nahezu mineralfreie Schieferkohlen, das heißt stark gepresste blättrige Lagen von Bruchwaldtorfen. Diese Sedimente dominieren vor allem im unteren Drittel des DA. So unterschiedlich wie die Sedimente ist auch die jeweilige Pollenführung, wobei keineswegs die humosesten Lagen auch die beste Pollenführung aufweisen.

$\mathrm{Zu}$ Beginn des DA bleibt das $\mathrm{BP} / \mathrm{NBP}-$ Verhältnis vom vorhergehenden Abschnitt annähernd erhalten (DA-Basis 97,3\% BP). Der BP nimmt dann aber nach oben hin mit einigen Schwankungen allmählich ab und weist am Ende des DA nur noch 88,3\% auf. Die Abnahme des Gehölzpollens resultiert aus dem Rückgang der EMW-Gehölze, von Abies und Corylus sowie dem Verschwinden von Carpinus. Diese Holzart kommt hier nur noch mit einem Pollenkorn im Spektrum $790 \mathrm{~cm}$ vor. Am Ende des DA enden alle Kurven thermophiler Holzarten, auch Picea geht mit ihren Werten zurück. Pinus dagegen bleibt nahezu unverändert.

Der Anteil von Almus, Pinus und Picea am Pollenniederschlag ist während des gesamten DA schwankend. Besonders die Alnus-Kurve hat mehrfach deutliche Spitzen und Einbrüche, am ruhigsten verläuft die Pinus-Kurve. Betula hat jetzt deutlich höhere Werte als im DA a und ihre Pollenkurve weist ebenfalls einige markante Gipfel auf. Am auffallendsten ist der Spitzenwert im Spektrum von $849 \mathrm{~cm}$ von $26,5 \%$, danach fällt die Kurve aber gleich wieder auf die vorherigen Werte um 10\% zurück. Erst gegen Ende des DA b steigt die Betula-Kurve parallel zu derjenigen der Kiefer wieder an und erreicht am Abschnittsende 


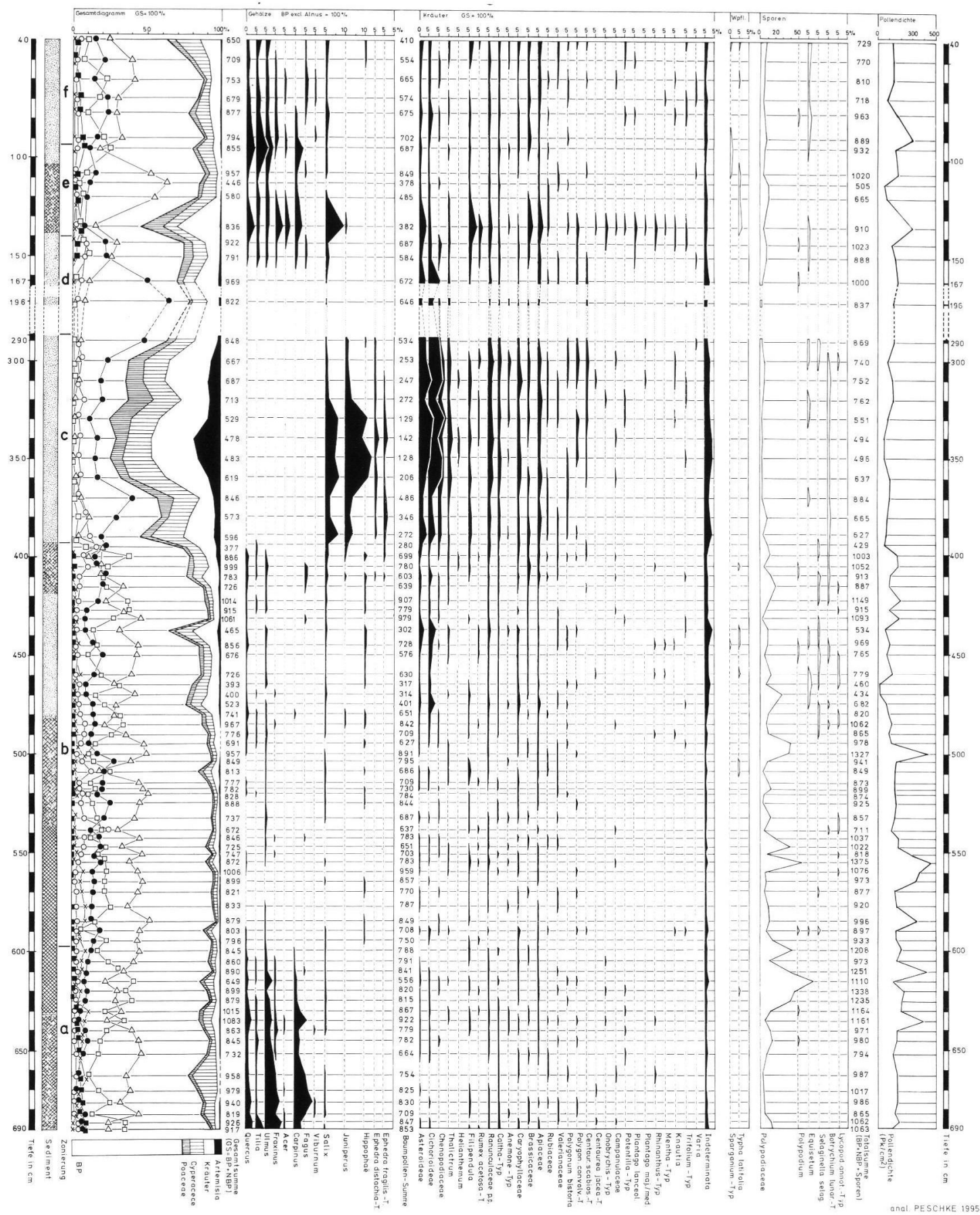

Abb. 6: Pollendiagramm der Kernbohrung von Unterhalb La Flachère nach der herkömmlichen Darstellungsweise $($ Peschke $)$. Grundsumme $=\mathrm{BP}+\mathrm{NBP}=100 \%$. Lithostratigraphie: Punkte $=$ Siltige Tone. Schräge Raster $=$ Schieferkohlen. Schräge Raster mit Punkt: Tonige Schieferkohlen. - Kartographie: P. PEschke, G. Koch.

Fig. 6: Pollen diagram of the drilling "Below La Flachère" after the common presentation form of (PESCHKE). Total sum $=\mathrm{AP}+\mathrm{NAP}=100 \%$. Lithostratigraphy: dots = silty clay; diagonal screen = slate coal; diagonal screen dotted = clayey slate coal. - Cartography: P. Peschke, G. KocH. 
19,5\% bei einem Pinus-Anteil von 25,7\%. An dieser Stelle sind keine thermophilen Gehölze mehr nachzuweisen, dafür beginnen die geschlossenen Pollenkurven von Juniperus und Ephedra. Außerdem ist jetzt Salix im Pollenniederschlag stärker vertreten. Diese sich in der Auflichtung des Waldes und dem Vorkommen heliophiler Gehölze niederschlagende Klimaverschlechterung zeigt sich auch in einer allgemeinen Zunahme der Kräuter, besonders von Artemisia, Chenopodiaceae und Asteraceae. Für die Erle müssen sich die Standortverhältnisse gravierend verschlechtert haben, möglicherweise auch Größe und Anzahl der Nassstandorte kleiner geworden sein. Die Amus-Pollenwerte liegen im gesamten DA b zwischen 20 und $40 \%$ und erreichen kur $\angle$ vor $\mathrm{Ab}$ schnittsende im Spektrum bei $710 \mathrm{~cm}$ den Spitzenwert von 43,8\%. Danach fällt die Pollenkurve dieser Holzart auf Werte von 5-10\% zurück, die langandauernde Dominanz des Erlenpollens in der unteren Diagrammhälfte wird nicht mehr erreicht. Dieser Rückgang kann nur mit einer geringeren Beteiligung dieser Holzart am Vegetationsbild erklärt werden.

Das Waldbild in diesem DA ist von der Fichte geprägt mit geringer Beteiligung von Tanne und Kiefer, dazu wenig EMW-Gehölze, meist Eiche und Ulme sowie etwas Haselnuss. Häufiges Auftreten der Erle spricht für ausgedehnte Bruchwald- und Auwaldstandorte im Talbereich. Auf offenen Stellen im Bereich der Isère kamen auch etwas Sanddorn und Weide vor.

Allgemein zeigt der Abschnitt b noch das Abbild einer bewaldeten Landschaft, denn der NBP-Anteil bleibt überwiegend unter 10\%. Nur wenn Süß- und Sauergräser mitberücksichtigt werden, gibt es ab und zu höhere NBP-Werte. Offene, das heißt waldfreie Flächen können im Bereich des verwilderten Isère-Flusssystems vorgekommen sein, z. B. auf Kiesbänken oder Sandalluvionen oder auch auf steilen und exponierten Partien der Talflanken, wo Felsen aus dem Waldland herausragten und nur einer Gräser-Kräuter-Vegetation besiedelbare Standorte boten.

\section{DA c: $605-700 \mathrm{~cm}$ Profiltiefe}

Das untersuchte Sediment dieses Abschnittes bestand durchweg aus grauen, siltigen Tonen mit geringen organischen Beimengungen und Molluskenresten. Die Pollenführung erwies sich als ähnlich gut wie in den tonigen Schieferkohlen mit ihrem hohen Anteil an organischer Substanz. Die Erhaltung war allerdings schlechter. Dies kommt in höheren Werten der Indeterminata zum Ausdruck.

Die sich am Ende des DA b ankündigenden klimatischen Verschlechterungen für den Pflanzenwuchs sind jetzt voll eingetreten: Die Gehölzvegetation hat deutlich abgenommen, ihr Pollenanteil sinkt unter $40 \%$ und erreicht im Spektrum bei $640 \mathrm{~cm}$ einen Tiefstand von 35,8\%. Thermophile Holzarten sind völlig verschwunden, lediglich $\mathrm{Al}$ mus ist noch mit geschlossener Kurve und Werten um 2-3\% vertreten. Bei Berücksichtigung der Überrepräsentation dieses Pollentyps kann aber die Baumart im Vegetationsbild keine große Rolle mehr gespielt haben. Den höchsten Anteil am BP nimmt Pinus ein, gefolgt von Betula und $J u$ niperus mit einem Maximum von 5,3\% im Spektrum bei $670 \mathrm{~cm}$. Picea ist weiterhin mit geschlossener Kurve vertreten, ihre Werte sinken aber bis unter 2\%. Salix und beide Ephedra-Typen vervollständigen das Arteninventar der Gehölze.

Die vom Wald verlassenen Standorte besiedelt jetzt eine reichhaltige Gräser-Kräuter-Pflanzengesellschaft, in welcher Artemisia eine dominierende Rolle zukommt mit einem Maximum ihrer Pollenkurve von 23,0\% im Spektrum bei $650 \mathrm{~cm}$. Neben den Süßgräsern sind gegenüber dem vorigen DA auch die Sauergräser stärker repräsentiert. Dies verweist auf eine Vergrößerung der Riedvegetation auf Sumpf- und Nassstandorten. Am Pollenniederschlag dieser Taxa werden aber auch alpine Seggen- und Süßgräserarten beteiligt gewesen sein, welche die jetzt mehr offenen terrestrischen Standorte der Talhänge besiedelt haben.

Das Vegetationsbild dieser Zeit war bestimmt von einem aufgelockerten Wald, der vorwiegend aus Kiefern und Birken mit etwas Fichte und Wacholder bestanden hat. Wahrscheinlich sind die Gehölze auf edaphisch günstigen Standorten in kleinen Inseln bzw. Gruppen vorgekommen. Erle und verschiedene Weidenarten können vereinzelt Fluss- und Bachufer begleitet haben. Nach den geringen Pollenwerten wird es aber kaum zu Galeriesäumen gekommen sein. Die offenen Standorte waren von einer artenreichen GräserKräuter-Vegetation bedeckt, die NBP-Typenzahl erreicht in diesem DA die höchsten Werte des gesamten Diagramms. Die konstante Beteiligung von Chenopodiaceae am Pollenniederschlag und das reichliche Vorkommen von Artemisia sind Ausdruck einer "Steppen-Tundra", wie sie in Mitteleuropa bei kaltzeitlichen Bedingungen im Laufe des Quartärs immer wieder vorkam. 


\section{DA d: 468,5 - 605 cm Profiltiefe}

Obwohl die Sedimente dieses Abschnittes in der Profilsäule über $1,3 \mathrm{~m}$ ausmachen, konnten daraus nur 3 Pollenspektren erarbeitet werden. Weite Bereiche der Profilstrecke waren pollenfrei oder enthielten nur vereinzelt Sporomorphen, die keine Auswertung erlaubten. Im Gegensatz zu vorher waren die graubläulichen siltigen Tone wesentlich kompakter gelagert, zerriebene Reste von Molluskenschalen waren noch zu erkennen, organische Beimengungen fehlten aber vollkommen. Wahrscheinlich haben sich die Klimaverhältnisse des DA c noch weiter verschlechtert und die Vegetation wurde stark dezimiert bzw. geschwächt, so dass die Pollenproduktion der wenigen Arten drastisch zurückging und der Pollenniederschlag sich verteilte, weshalb er kaum mehr fassbar ist.

Dieser Vermutung steht allerdings entgegen, dass sich in den drei auswertbaren Spektren bei 600, 506 und $477 \mathrm{~cm}$ eine bessere Klimasituation abzuzeichnen scheint als im DA c: Der BP und hier vor allem Pinus ist deutlich höher als zuvor und "Kältezeiger" unter den Gehölzen fehlen. Der NBP ist dabei aber annähernd so artenreich wie vorher, nur der Anteil der Gräser ist etwas zurückgegangen. Diese drei Spektren liegen in dieser sonst mehr oder weniger pollenfreien Profilstrecke allerdings sehr isoliert und weit auseinander, so dass sie kaum Ausdruck einer zusammenhängenden Vegetationsentwicklung sein können. Sehr wahrscheinlich sind diese Spektren durch umgelagerte Pollenkörner verfälscht, auch wenn dabei kein Thermophilpollen gefunden werden konnte. Damit entfällt auch die Möglichkeit, aus den Pollenfunden dieses Abschnittes ein Vegetationsbild zu rekonstruieren. Die hier sedimentierten, überwiegend sporomorphenfreien Tone müssen aus einem fast vegetationsfreien Zeitraum stammen und Ausdruck eines sehr kalten Klimas sein.

\section{DA e: 383 - $468,5 \mathrm{~cm}$ Profiltiefe}

Die Sedimente dieses Diagrammabschnittes weisen in den grauen Tonen an der Basis, die von schwarzbraunen kompakten Schieferkohlen überlagert werden, wieder höhere organische Anteile in Form von Holzresten und Schieferkohlenspuren auf. Die obersten Horizonte von DA e werden erneut von grauen sandig-tonigen Ablagerungen mit organischen Beimengungen gebildet. Die Pollenführung dieses Abschnittes ist wechselhaft, gute Spektren sind mehrfach von mehr oder weniger pollenfreien Straten unterbro- chen, was zu Lücken im Diagramm führte. In den Schieferkohlen ist die Pollenerhaltung mittelmäßig und die Pollenbeobachtung häufig durch Überlagerung insbesondere durch Gewebebruchstücke erschwert, wie dies in derartig detritusreichen Rückständen aus Schieferkohlen üblich ist. Hier bewährte sich bei der Analyse erneut das Auszählen in Glycerin, wobei die Suspension unter dem Deckglas etwas bewegt werden kann und verdeckte Pollenkörner oft frei werden, die dann sicherer angesprochen werden können. Der Baumpollen hat jetzt wieder deutlich zugenommen und erreicht Werte über $90 \%$, mit einem Maximum von 96,4\% im Spektrum bei $420 \mathrm{~cm}$. Picea ist die dominierende Holzart und erreicht in diesem Diagramm mit 71,6\% im Spektrum bei $422 \mathrm{~cm}$ den höchsten Wert überhaupt. Pinus hat an Bedeutung verloren, Almus und Betula dagegen gewonnen. Außerdem treten wieder Abies und Carpinus sowie alle EMW-Gehölze mit Corylus auf und deuten eine ähnliche Waldzusammensetzung an, wie sie vor der Kältephase von DA d und c im DA b bzw. am Ende des DA a geherrscht hatte. Der Kräuterpollen ist in der unteren Hälfte des DA e noch verhältnismäßig artenreich, auch wenn hier dickwandige und stark strukturierte Pollentypen wie die der Asteraceae überrepräsentiert sind.

Im mittleren Teil von DA e lässt sich zwischen 420 $\mathrm{cm}$ und $430 \mathrm{~cm}$ eine Klimaverschlechterung erkennen, die sich in einer sprunghaften Zunahme der Kiefer und einem Rückgang der Thermophilen niederschlägt.

Die im DA e gefundene Pollenflora vermittelt das Bild eines Nadelwaldes mit eingestreuten anspruchsvollen Laubgehölzen, wie es im Alpenraum einschließlich der Vorländer interstadialen Zeiträumen zugeordnet wird. Die wärmeliebenden Gehölze werden vermutlich in erster Linie auf kalkigem Untergrund die SE exponierte Talflanke der Chartreuse besiedelt haben. Die häufige Fichte wird einerseits auf den Oberhängen und in den Hochlagen der kristallinen Belledonne vermutet, andererseits aber auch auf vernässten Standorten des Talbodens, dort in Gesellschaft von Erle und Weidenarten. Ausgesprochen heliophile Gehölz- und Kräuterarten fehlen, so dass der Waldbestand ziemlich geschlossen gewesen sein muss.

\section{DA f: $\mathbf{3 5 0}-\mathbf{3 8 3} \mathbf{c m}$ Profiltiefe}

Das Sediment dieses Abschnittes bestand aus graublauen Tonen mit organischen Beimengungen unterschiedlicher Größe, teilweise auch 
Holzresten. Stellenweise waren feine horizontale Schichten vermutlich eingeschwemmten organischen Materials zu erkennen. Die Pollenführung war zufriedenstellend, die Erhaltung mäßig.

Die Pollenflora hat vor allem Ähnlichkeit mit dem kurzen kühleren Abschnitt von DA e zwischen $420 \mathrm{~cm}$ und $430 \mathrm{~cm}$. Pinus ist die vorherrschende Baumart. Der BP schwankt um 90\% und bei den Gehölzen verschwindet Carpinus, die bereits in der Mitte von DA e zurückgeht. Im DA f fällt auch Abies unter die 1\%-Grenze und fehlt im Spektrum bei $360 \mathrm{~cm}$ vollkommen. Die EMW-Gehölze und Corylus sind insgesamt schwächer vertreten als im liegenden Diagrammabschnitt. Die thermophile Begleitflora des Fichten-Kiefernwaldes ist im DA f also etwas ärmer als im DA e. Ob dies auf klimatische Veränderungen zurückgeführt werden darf, ist nicht eindeutig, denn Kälteanzeiger in der Gehölzflora oder Zeiger für offene Vegetation bei den Kräutern wie Artemisia und anderen Heliophyten sind auch nicht zahlreicher als zuvor. Anstelle von Carpinus kommt im ganzen Abschnitt jetzt Fagus mit Werten um 1\% vor, die sich im vorigen DA schon in drei Spektren angekündigt hatte. Vielleicht lässt sich hieraus eine etwas größere Humidität des Klimas ableiten. Alnus bleibt bei ähnlich hohen Werten wie am Ende des DA. An der Größe des Nassstandortes dürfte sich nichı viel verändert haben.

Die oberen $40 \mathrm{~cm}$ anthropogen umgelagerten Sediments bis zur Geländeoberkante des Bohransatzpunktes konnten nicht palynologisch bearbeitet werden. Die Fortsetzung der Ablagerungen nach oben wurde bereits früher wenige Meter hangwärts ergraben und das Profil von $350 \mathrm{~cm}$ Länge schon zweimal bearbeitet, und zwar von BotTema \& Koster (in: Hannss et al. 1976: 501 ff.) und Gremmen (1982: Diagr. 10, 27, 44, 45). Dabei lässt sich die Basis der Pollenzone DA 1 von GREMmen zwanglos an den hier beschriebenen DA $\mathrm{f}$ anschließen und verlängert die Fichten-KiefernNadelwaldphase beträchtlich (Abb. 7).

GREMmen (1982: 44, 45) beschrieb seine 5 Pollenzonen folgendermaßen:

\section{DA 1: $293-350 \mathrm{~cm}$}

Hier (Spektren 1-3) dominieren abwechselnd Pinus- und Picea-Pollen. Amus und Corylus sind gut vertreten. Tilia-Pollenkörner wurden mit 5\% gefunden, Ulmus, Quercus und Abies kommen nur in sehr geringen Prozentsätzen vor. Die NAPWerte sind niedrig, Dryopteris-Sporen machen hohe Anteile aus.

\section{DA 2: $183,5-293 \mathrm{~cm}$}

In diesem Abschnitt 2 (Spektren 4-12) dominiert der Picea-Pollen und stellt zusammen mit dem von Pimus den Hauptteil des AP-Pollens. Der Alnus-Anteil ist im allgemeinen niedrig, ausgenommen das über $40 \%$ erreichende Maximum in Spektrum 5. Unter den anderen Pollentypen sind Tilia und Quercus am besten repräsentiert, aber beide nur mit niedrigen Werten. Der NAP-Pollen macht lediglich einige wenige Prozente aus. Dryopteris kommt sehr wenig vor.

\section{Da 3: $160-183,5 \mathrm{~cm}$}

In dieser Pollenzone 3 (Spektren 13 und 14) dominiert Pinus mit rund $40 \%$. Picea-Pollen ist ebenfalls mit eher hohen Werten vertreten. Alnus ist gut repräsentiert. Von den anderen Bäumen erreicht nur Betula mehr als 5\%. Der Anteil von Abies ist schwach, der des NAP-Pollen geht nicht über 13\% hinaus. Artemisia ist am stärksten verbreitet. Cyperaceae und Gramineae weisen hohe Werte auf. Dryopteris ist eher gut vertreten.

\section{DA 4: $134,5-160 \mathrm{~cm}$}

Hier (Spektren 15-16) sind die NAP-Werte niedrig. Picea nimmt zu und wird zum beherrschenden Pollentyp. Laubabwerfende Bäume sind schwach vertreten, abgesehen von Alnus und Betula; Gramineae- und Cyperaceae-Pollenkörner zeigen einen starken Rückgang. Die DryopterisWerte sind sehr niedrig.

\section{DA 5: $2-134,5 \mathrm{~cm}$}

In diesem Diagrammabschnitt (Spektren 17-23) geht die Picea-Kurve auf unter $20 \%$ zurück. Die Pinus-Werte nehmen von $40 \%$ auf $70 \%$ zu. Abies fehlt in dieser Zone. Pollen laubabwerfender Bäume hat keinerlei Bedeutung. Betula und Salix kommen vor, aber nur mit geringen Prozentanteilen. Verschiedene Pollentypen wie Artemisia, Chenopodiaceae, Compositae, Tubiliflorae, Umbelliferae und Thalictrum tragen zu hohen NAPWerten in der Pollenflora bei. Insgesamt sind die Prozentanteile von Gramineae eher niedrig, die von Cyperaceae eher hoch. Die Maximalwerte werden jeweils in den Spektren 22 und 23 erreicht.

Das teilweise schon recht kühle Klima, das während der Ablagerung des aufgeschlossenen Profils von Unterhalb La Flachère geherrscht hat, schlägt sich auch in den Funden von zwei Stücken Birkenholz, ein bis drei Stücken Fichtenholz und einem weiteren fossilen Nadelholz nieder (Bestimmung durch F. SchweInGruber in Hannss 1982: Tab. 6). 


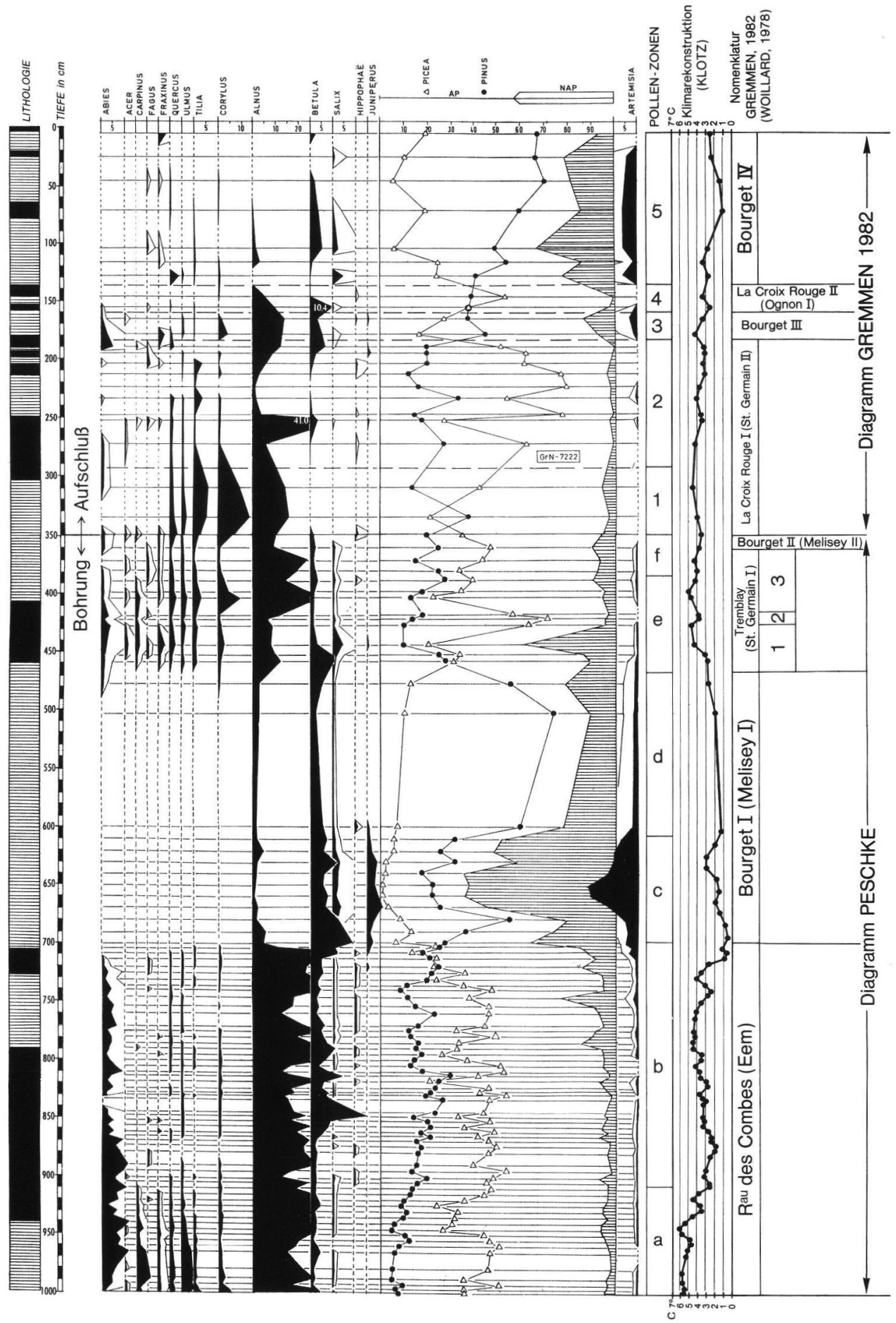

Abb. 7: Gesamtpollendiagramm von Unterhalb La Flachère nach der Darstellungsweise von GremmEN (1982). Waagrechte Striche $=$ fossilführende tonige Silte. Schwarz $=$ Schieferkohlen. Grundsumme $=$ BP + NBP ohne Gramineae und Poaceae. - Kartographie: P. Peschke, G. Koch.

Fig. 7: Total pollen diagram from "Below La Flachère" after the presentation form of GREMMEN (1982).

Total sum $=\mathrm{AP}+\mathrm{NAP}$ without Gramineae and Poaceae. Lithostratigraphy: horizontal lines = fossiliferous clayey silts; black = slate coal. - Cartography: P. PeschKE, G. KoCH. 


\section{Die biostratigraphische Einstufung}

Das aus der Kernbohrung erarbeitete Pollendiagramm (Abb. 5 4), Abb. 65 ) zeigt an der Basis (DA a und b) eindeutig eine warmzeitliche Pollenflora eines Fichten-Tannenwaldes mit nach unten zunehmender Beteiligung thermophiler Laubgehölze. Diese deuten die Endphase eines interglazialen Laubwaldoptimums an. Leider ist dieser Zeitabschnitt und die Einwanderung der wärmeliebenden Gehölzflora nicht mehr überliefert. Dies erschwert den Vergleich mit anderen kompletten Pollendiagrammen interglazialer Ablagerungen. Zum Ende der Warmzeit (DA b) nehmen die Thermophilgehölze wie die Tanne allmählich ab. Ein deutlicher Kiefern-Birken-Vorstoß bei Rückgang der Fichte leitet danach zu einer ausgeprägten Kaltphase (DA c) über, in welcher viel Kräuterpollen mit Artemisia sowie Wacholder die Pollenspektren prägen. Über dieser Kaltphase liegen Silte mit hohem Tonanteil, die nur in wenigen Spektren und großen Probenabständen eine auswertbare Pollenflora enthielten (DA d). Diese Sedimente müssen noch am Ende der Kaltphase abgelagert worden sein. Die Pollenakkumulation während dieser Phase war nur gering, weil auf Grund der offenen kaltzeitlichen und lockeren Vegetationsdecke nur vergleichsweise wenig Pollen produziert worden ist.

Nach dieser Kaltphase breitet sich erneut die Waldvegetation aus, woran auch EMW-Gehölze und die Hasel beteiligt sind. Tanne und Hainbuche sind jetzt deutlich geringer vertreten als in den unteren stadialen Abschnitten. Die relativ hohe Beteiligung von Fichte und Kiefer zeigt an, dass während der Waldzeit DA e, die durch eine Abkühlungsphase zweigeteilt ist, ein interstadialzeitlicher Klimacharakter vorgeherrscht hat. Der DA f spiegelt im Vergleich hiermit kühlere Klimabedingungen wider. Somit ist in den erbohrten Sedimenten eine ausklingende Warmzeit abgebildet, die über eine ausgeprägte Kaltphase zu einer interstadialen, wahrscheinlich zweigeteilten Waldvegetationsperiode mit wenigen thermophilen Laubgehölzen überleitet, auf die nach oben hin eine weitere Abkühlungsphase folgt.

Das von GRFmmen erarbeitete Pollendiagramm von Unterhalb La Flachère (1982: Diagr. 10), das an einst aufgeschlossenen Schieferkohlenführenden Feinablagerungen gewonnen worden

4) Nach der Diagrammdarstellung von GREMmEN (1982: Diag. 10)

5) In herkömmlicher Darstellung mit Gesamtdiagramm und Baumpollendiagramm ohne Almus. ist, schließt sich augenscheinlich nahtlos an die hier 1991 erbohrte Sequenz an. Im DA 1 und 2 (Abb. 7) folgt auf ein eher boreal getöntes Interstadial mit wenig Thermophilen in DA 3 eine deutliche stadiale Abkühlungsphase, während der die Kiefer über die Fichte dominierte, und im DA 4 eine interstadialzeitliche Wiedererwärmung, die durch ein Überhandnehmen der Fichte über die Kiefer und das Fehlen thermophiler Laubbäume gekennzeichnet ist. Darüber zeichnet die Pollenflora im DA 5 eine weitere, von der Kiefer dominierte stadiale Abkühlungsphase nach, wobei die beiden obersten Spektren eine leichte Klimaverbesserung in ein oberstes Fichten-Kiefern-Interstadial hinein andeuten könnten.

Bei dem Versuch einer zeitlichen Einstufung dieser Ablagerungen kommen als Vergleichsdaten in erster Linie die zahlreichen Pollendiagramme von GREMmEN (1982) aus dem nahegelegenen Val du Bourget und dem Grésivaudan selbst in Frage. Daneben können zum weiteren Vergleich mit der Sequenz von Unterhalb La Flachère noch zwei nicht zu weit entfernt liegende lange Pollensequenzen aus dem Alpenvorland herangezogen werden. Es handelt sich dabei zum einen um die pollenanalytisch bearbeitete Abfolge von Les Echets (De Beaulieu \& ReIlle 1984) aus den östlich von Lyon gelegenen Dombes und zum anderen um die der Schieferkohlen von Gondiswil im Schweizer Mittelland (Wegmüller 1992). Sie spiegeln jedoch etwas andere Vegetationsverhältnisse wider, insbesondere hinsichtlich der Gebirgsbaumarten Fichte und Tanne. Die wesentlichen Veränderungen der Klimaverhältnisse zeichnen sich aber hier wie dort ab. Die entsprechenden Diagramme zeigen in Les Echets wie in Gondiswil die Abfolge eines an Thermophilen reichen Interglazials. Während in Les Echets darüber noch zwei weitere Warmphasen gleichen Charakters entwickelt sind, folgen in Gondiswil zwei boreale Interstadiale, in denen die thermophilen Laubbäume noch spärlicher vertreten sind als in den Schieferkohle-führenden Feinablagerungen von Unterhalb La Flachère. Darüber zeichnen sich weitere kühlere, allein durch die Fichte und Kiefer charakterisierte Interstadialphasen ab.

An beiden Lokalitäten ist im unteren Teil der Ablagerungen das komplette letzte Interglazial und die Einwanderung der verschiedenen thermophilen Holzarten erfasst. Ihre jeweilige Ausbreitung zeichnet sich gut ab. Diese Kriterien sind für das Unterhalb La Flachère-Diagramm leider nicht nutzbar. Erst im jüngeren Teil des Interglazials, nach dem EMW- und Taxus-Maximum, ist in der 
Tannen-Fichten-Hainbuchen-Phase eine Vergleichsmöglichkeit gegeben, ebenso bei dem dann erfolgenden Wechsel zur ersten Kaltphase und dem sich anschließenden Interstadial.

Diagrammvergleiche mit weiter entfernten Lokalitäten im östlichen Alpenvorland oder gar in Norddeutschland erscheinen wenig sinnvoll, da über die Areale waldbestimmender Baumarten in älteren Warmzeiten noch viel zu wenig bekannt ist. Diese waren sicher nicht mit den heutigen Arealen vergleichbar. Auch weiß man wenig über die Einwanderungsbahnen der einzelnen Holzarten, die in älteren Warmzeiten nicht so wie im Postglazial verlaufen sein müssen.

Hinzu kommt, dass Lokalitäten außerhalb der Alpen durch ihre gemäßigtere Topographie den Waldgesellschaften einheitlichere und oft auch großflächigere Standorte geboten haben als im stärker reliefierten Gebirgsinnern, wo eine Vielzahl extremer Standorte mit unterschiedlicher Exposition, Höhenlage und Böden ein viel reichhaltigeres Vegetationsmosaik zulässt. Durch die Reliefunterschiede werden hier auch die klimatischen Verhältnisse sowie die Pollenverwehung und -einschwemmung und damit die Pollensedimentation stärker beeinflusst als im Vorland. So ist im Gebirge der Eintrag von Kräuterpollen aus der baumfreien alpinen Vegetation bestimmt deutlicher als im Vorland - und in Kaltzeiten

Tab 3: Korrelation der Chronozonen von Gremmen (1982: Fig. 14), WollLARD (1978: Fig. 2) und der Isotopenstadien (OIS).

Tab. 3: Correlation of the chrono zones of Gremmen (1982, Fig.14), WoilLARD (1978, Fig. 2) and the isotopic stages (OIS).

\begin{tabular}{|l|l|l|}
\hline \multicolumn{1}{|c|}{ GREMMEN } & \multicolumn{1}{|c|}{ WOILLARD } & \multicolumn{1}{c|}{ Isotopenst. (OIS) } \\
\hline $\begin{array}{l}\text { Frühestweichsel } \\
\text { Sonnaz II }\end{array}$ & Lanterne II & $5 \mathrm{a}-4$ \\
\hline Tillet II & Lanterne II & $5 \mathrm{a}-4$ \\
\hline Sonnaz I & Lanterne II & $5 \mathrm{a}-4$ \\
\hline Tillet I & Lanterne II & $5 \mathrm{a}-4$ \\
\hline La Croix Rouge III & Ognon II & $5 \mathrm{a}-4$ \\
\hline Bourget IV & Stade Ognon II & $5 \mathrm{a}-4$ \\
\hline La Croix Rouge II & Ognon I & $5 \mathrm{a}-4$ \\
\hline Bourget III & Stade Ognon I & $5 \mathrm{a}-4$ \\
\hline $\begin{array}{l}\text { Letztes Interglazial: } \\
\text { La Croix Rouge I }\end{array}$ & St. Germain II & $5 \mathrm{a}$ \\
\hline Bourget II & Melisey II & \\
\hline Tremblay & St. Germain I & $5 \mathrm{c}$ \\
\hline Bourget I & Melisey I & \\
\hline Ruisseau des Combes & Eem & $5 \mathrm{e}$ \\
\hline Moulins & Linexert & 6 \\
\hline
\end{tabular}

steigt im Gebirge der NBP früher an, weil die Waldgrenze hier früher absinkt. All das kann regionale Unterschiede in zeitgleichen Pollendiagrammen einer Region erklären.

Vor dem Hintergrund seiner Befunde aus dem Val du Bourget korrelierte GREMmEN seinen obersten Profilabschnitt von Unterhalb La Flachère mit dem Bourget IV-Stadial, das nach der Einteilung und Terminologie der Grande Pile (WoIllard 1975, 1978) mit dem Ognon II-Stadial von WoILLARD (1978: 5) gleichzusetzen ist (Tab. 3). Nach unten folgt das von Kiefern und Fichten bestimmte La Croix-Rouge II-Interstadial, das wahrscheinlich mit dem Ognon I-Interstadial zusammenfällt. Abgetrennt von dem Bourget III-Stadial, dem Ognon I-Stadial von Wolllard (1978: 5), schließt sich nach unten das La Croix-Rouge I-Interstadial an, welches GREMmen mit dem warmzeitlichen St. Germain II-Interstadial gleichgesetzt hat. Es wird nach unten von dem Bourget II-Stadial begrenzt, das dem Melisey II-Stadial von Wonllard entspricht. Folgerichtig ist darunter dann das Tremblay-Interstadial entwickelt, das GREMMEN mit dem dreigeteilten und ebenfalls warmzeitlichen St. Germain I gleichgesetzt hat. In seinen Verlauf waren die thermophilen Laubbäume im Val du Bourget weniger stark verbreitet als während des La Croix Rouge I-Interstadials, also dem St. Germain II (Gremmen 1982: Diagr. 1, 2, 6; Gremmen \& Hannss 1994: Fig. 4). Das sich unter dem TremblayInterstadial abzeichnende Bourget I-Stadial muss dann mit dem Melisey I gleichgesetzt werden und darunter die jüngere Hälfte des Rau des Combes-Interglazials und damit des oberen Eems entwickelt sein.

An der hier vorgeschlagenen bzw. von GREMMEN übernommenen Gliederung des Pollendiagramms von Unterhalb La Flachère bleiben besonders im mittleren und oberen Teil Zweifel bestehen, und zwar deshalb, weil nach oben hin die Sedimentationsraten augenscheinlich stark abgenommen haben und einige klimastratigraphische Einheiten nur 
durch ganz wenige Pollenspektren repräsentiert sind. Insbesondere gilt das für das Tremblay 2 sowie für das Bourget II- und Bourget III-Stadial.

Trotz dieser Unsicherheiten ist jedoch davon auszugehen, dass das zusammengefasste Diagramm der Bohrung und des Aufschlusses von Unterhalb La Flachère (Abb. 7) das ganze letzte Interglazial zeigt, dessen frühe und mittlere Phasen wie bei den anderen Pollensequenzen gleichen Alters aus dem Val du Bourget und dem Grésivaudan (Gremmen 1982: Diagr. 1-3, 9; Gremmen \& Hannss 1994: Fig. 4) aber auch hier nicht abgebildet sind. Darüber sind noch zwei bereits wesentlich borealer gefärbte Interstadiale entwickelt, in denen die Thermophilen keine allzu große Rolle mehr spielten, sowie ein frühestweichselzeitliches Interstadial, in dem sie so gut wie gar nicht mehr vertreten gewesen sind. In diesem dominieren eindeutig die Kiefer und die Fichte. Darüber ist mindestens noch ein zweites frühestweichselzeitliches Kiefern-Fichten-Interstadial (Abb. 3, 4) in einer rund $50 \mathrm{~cm}$ mächtigen, tonig-siltigen Sequenz entwickelt, die in rund $348 \mathrm{~m}$ ansteht und pflanzliche Reste enthält. Sie wird von nach unten hin konglomeratisierten Schottern überlagert, die nach oben in relativ grobe Vorstoßschotter übergehen, deren Feinsedimentlinsen sich jedoch schon als pollenleer erwiesen haben (Abb. 3).

Die bisher für den Schieferkohlen-Aufschluss von Unterhalb La Flachère gewonnenen Radiokarbonmindestalter von $>21100,>35000,>45000$ und $>56400$ stehen in keinem Widerspruch zu der vorgeschlagenen biostratigraphischen Einstufung. Das von GroOTEs erzielte Anreicherungsabsolutalter von $65000-1400 \pm 1700$ BP muss jedoch wohl eher als Mindestalter interpretiert werden (Tab. 2).

\section{Klimarekonstruktion (STEFAN KLOTZ)}

Fossile Pollenspektren spiegeln nicht nur in gewissem Maße die regionale Paläovegetation wider, sondern geben aufgrund der Florenzusammensetzung und ökologischen Ansprüche der einzelnen Pflanzen auch Auskunft über die regionalen klimatischen Verhältnisse. Mittels quantitativer Rekonstruktionsmethoden lässt sich danach zu fossilen Spektren das Paläoklima rekonstruieren, so dass bei Anwendung auf die gesamte Pollenchronik die Klimaentwicklung des betrachteten Zeitabschnitts aufgezeigt werden kann.

Den frühen Ansatz eines quantitativen Rekonstruktionsverfahrens zeigt die Indikatorartmethode von IVERSEN, bei der die Verbreitung von Vis- cum, Hedera und Ilex in Abhängigkeit von der mittleren Temperatur des wärmsten (MTWM) und des kältesten Monats (MTKM) untersucht wurde, so dass für alle Kombinationen der genannten Pflanzen klimatische Aussagen möglich waren, insbesondere bei Vorkommen in quartären Spektren (IVERSEN 1944: 463-483). In ähnlicher Weise beschreibt GRICHUK die klimatische Verbreitung zahlreicher Bäume, indem deren heutiges Vorkommen in Abhängigkeit von der MTWM und MTKM in einem Diagramm dargestellt wird, um deren klimatische Existenzgebiete abzugrenzen. Das Existenzgebiet einer fossilen Flora kann dann dadurch rekonstruiert werden, dass die gemeinsame Überschneidung der Existenzgebiete der nächsten lebenden Verwandten zu den fossilen Pflanzen bestimmt wird (GRICHUK 1969: 41-57; GrichuK et al. 1984: 251-260). Für Anwendungen im Tertiär zeigt die Koexistenzmethode genaue Werte, bei der die Verbreitung der Pflanzen jeweils nur in Abhängigkeit eines Klimafaktors betrachtet wird (Mosbrugger \& Utescher 1997: 6186). Klimarekonstruktionen für das Quartär werden heute hauptsächlich mit den Methoden der modernen Analoge durchgeführt, die mit Hilfe rezenter Oberflächenspektren und Klimadaten das Paläoklima ermitteln (GuIot et al. 1993: 73-93). Hierbei ist die zuerst benutzte Technik der Transferfunktionen zu nennen, die als multivariates statistisches Verfahren den funktionalen Zusammenhang zwischen Taxaprozentwerten und einzelnen Klimafaktoren herstellt (Iмbrie \& KIPP 1971: 71-147; Howe \& Webi III 1983: 17-51). Die pollen-climate response surfaces dagegen erlauben die Betrachtung der Florenverbreitung in Abhängigkeit mehrerer klimatischer Faktoren bei gleichzeitig anschaulicher Verbreitungsdarstellung (BARTLEIN et al. 1986: 35-57; HuntLey 1992: 91-99). Weiterhin ist die Methode der modernen Analoge mit paläobioklimatischem Operator nach Guiot anzuführen, bei der durch den Operator eine Gewichtung der klimatisch aussagekräftigeren Pflanzenarten erfolgt. Dabei wird zuerst zu jedem fossilen Pollenspektrum in Abhängigkeit von einem Distanzmaß eine bestimmte Anzahl dazu ähnlicher rezenter Oberflächenspektren ermittelt. Anhand der zu den Entnahmestellen der Oberflächenspektren zur Verfügung stehenden Klimawerte wird schließlich das Paläoklima berechnet, etwa durch Mittelung der einzelnen Klimawerte (Guıт 1987: 100-118; Guıт et al. 1989: 309-313).

Aufgrund der Vorteile der Berücksichtigung der Taxaprozentwerte in den Pollenspektren und der 
Gewichtung klimarelevanter Pflanzen bei der Methode der modernen Analoge mit paläobioklimatischem Operator, wurde dieses Verfahren im folgenden zur Klimarekonstruktion herangezogen. Da als Distanzmaß die logarithmierte euklidische Distanz benutzt wurde, werden bei der Auswahl der modernen Analoge zu einem fossilen Spektrum besonders Taxa mit geringen Anteilswerten berücksichtigt. Andererseits bedeutet dies, dass auch umgelagerte Pollenkörner größeren Einfluss auf die Klimarekonstruktion haben können. Die Datenbank der rezenten Oberflächenspektren enthält dabei Spektren neueren Datums von Guiot, Huntley und Prentice sowie älteren Datums von Birks und PETERSON, so dass der gesamte Datensatz 1327 rezente Oberflächenspektren, hauptsächlich aus dem europäischen Raum umfasst. Die einzelnen Pollenspektren setzen sich dabei jeweils aus den gleichen 43 Taxa zusammen, die vornehmlich Bäume, Sträucher und in geringer Anzahl Gräser und Kräuter repräsentieren (Guiot 1990: 49-69; Peyron et al. 1998: 183-196).

Der mittels der Methode der modernen Analoge rekonstruierte Verlauf der Klimaentwicklung wird hier mit der biostratigraphischen Zonierung von GREMmen in Bezug gesetzt, zumal dadurch die einzelnen Klimaphasen deutlicher herausgehoben und Übereinstimmungen aufgezeigt werden können. Der Verlauf der mittleren Jahrestemperatur (MJT) mit jeweils gemittelten benachbarten Werten ist im Gesamtpollendiagramm dargestellt und gibt einen ersten Überblick über die klimatische Entwicklung (Abb. 7). Die genaue Klimarekonstruktion mit allen Temperaturfaktoren sowie den Niederschlagswerten und der daraus resultierenden klimatischen Zonierung ist getrennt davon angegeben (Abb. 8).

Im DA a der Abb. 8 zeigt sich, ausgehend von einer mittleren Jahrestemperatur (MJT) von etwas über $6^{\circ} \mathrm{C}$ bei gleichzeitig höherer Ozeanität, ersichtlich an der MTWM um $16^{\circ} \mathrm{C}$ und der MTKM um $-5^{\circ} \mathrm{C}$ sowie hohen Niederschlagsmengen, eine im weiteren Verlauf des DA zunehmende $\mathrm{Ab}$ kühlung mit kontinentalerem Klima. Dabei sinkt die MJT bis auf $3^{\circ} \mathrm{C}$ und die MTKM auf $-10^{\circ} \mathrm{C}$ bei zudem abnehmenden Niederschlagsmengen. Ausdruck dieser Entwicklung sind die im allgemeinen geringer werdenden Taxawerte der bisher noch deutlich vertretenen thermophilen Gehölze sowie die Zunahme von Pinus.

Während sich zu Beginn des DA b die Abkühlung und eine starke Zunahme von Betula fortsetzen und den weiteren Rückgang thermophiler Gehölze bedingt, macht sich ab Mitte des DA b wiederum stärker ozeanisches Klima bemerkbar, wenn auch mit geringerer MJT und MTKM als noch zu Beginn des DA a. Die für den Beginn des DA a rekonstruierten Temperaturen liegen bei der MJT und MTKM etwa $4^{\circ} \mathrm{C}$ und bei der MTWM etwa $3^{\circ} \mathrm{C}$ unter denen der heutigen Messwerte im Untersuchungsgebiet (MülLER 1980: 11-21, 116125).

Neben der biostratigraphischen Zuordnung kann daraus geschlossen werden, dass es sich bei den besagten Abschnitten tatsächlich um das in Schüben kühler werdende "End-Eem" handelt. Entsprechende klimatische Verhältnisse zeigen sich trotz unterschiedlicher Vegetationszusammensetzung etwa auch für das Profil von Grande Pile in den südwestlichen Vogesen (GUIOT 1990: 63). Der hohe Nadelwaldanteil in Verbindung mit dem Auftreten thermophiler Gehölze in den DA a und $b$ bei Unterhalb La Flachère kann dabei durchaus lokalen hypsometrischen Einflüssen zugerechnet werden (Frenzel 1991: 51-78).

Infolge des abrupten Klimarückschlags im DA c mit einer MJT zwischen $-0,5^{\circ} \mathrm{C}$ und $3,5^{\circ} \mathrm{C}$, einer MTWM um $17,5^{\circ} \mathrm{C}$ und einer MTKM zwischen $10,2^{\circ} \mathrm{C}$ und $-15,4^{\circ} \mathrm{C}$ werden bei gleichzeitig geringeren Niederschlagswerten ausgeprägt kontinentale Verhältnisse angezeigt, wie sie heute etwa in der finnischen Region von Oulu und der anschließenden Region Karelien im NW Russlands vorzufinden sind. Damit können sowohl der DA c als auch der etwas wärmer erscheinende DA d, die jedoch beide durch hohe NBP-Werte zu charakterisieren und gegenüber dem „EndEem" leicht abzugrenzen sind, in das Bourget IStadial bzw. Melisey I-Stadial nach der im folgenden verwendeten WoILLARID-Terminologie gestellt werden. Die Vegetation entspricht dabei einer artenreichen Gräser-Kräuter-Steppe mit vereinzelten Gehölzelementen, wie sie vornehmlich auch für weite Gebiete Mittel- und Osteuropas nachgewiesen wurden (Frenzel 1968: 250). Ein klimatischer Vergleich mit Rekonstruktionen anhand von Käfern aus dem nördlichen Mitteleuropa ergibt für Unterhalb La Flachère eine bis zu $6^{\circ} \mathrm{C}$ höhere MTWM (WALKLING \& COOPE 1996: 145-159; CASPERS \& Freund 1997: 201-249). Dies wird sehr deutlich durch die artenreichere Vegetation und das Auftreten von Gehölzen getragen. Hingegen entspricht die MJT den für das Profil von Grande Pile rekonstruierten Werten (Guiot et al. 1989: 309-313). 
--- heutige Klimawerte

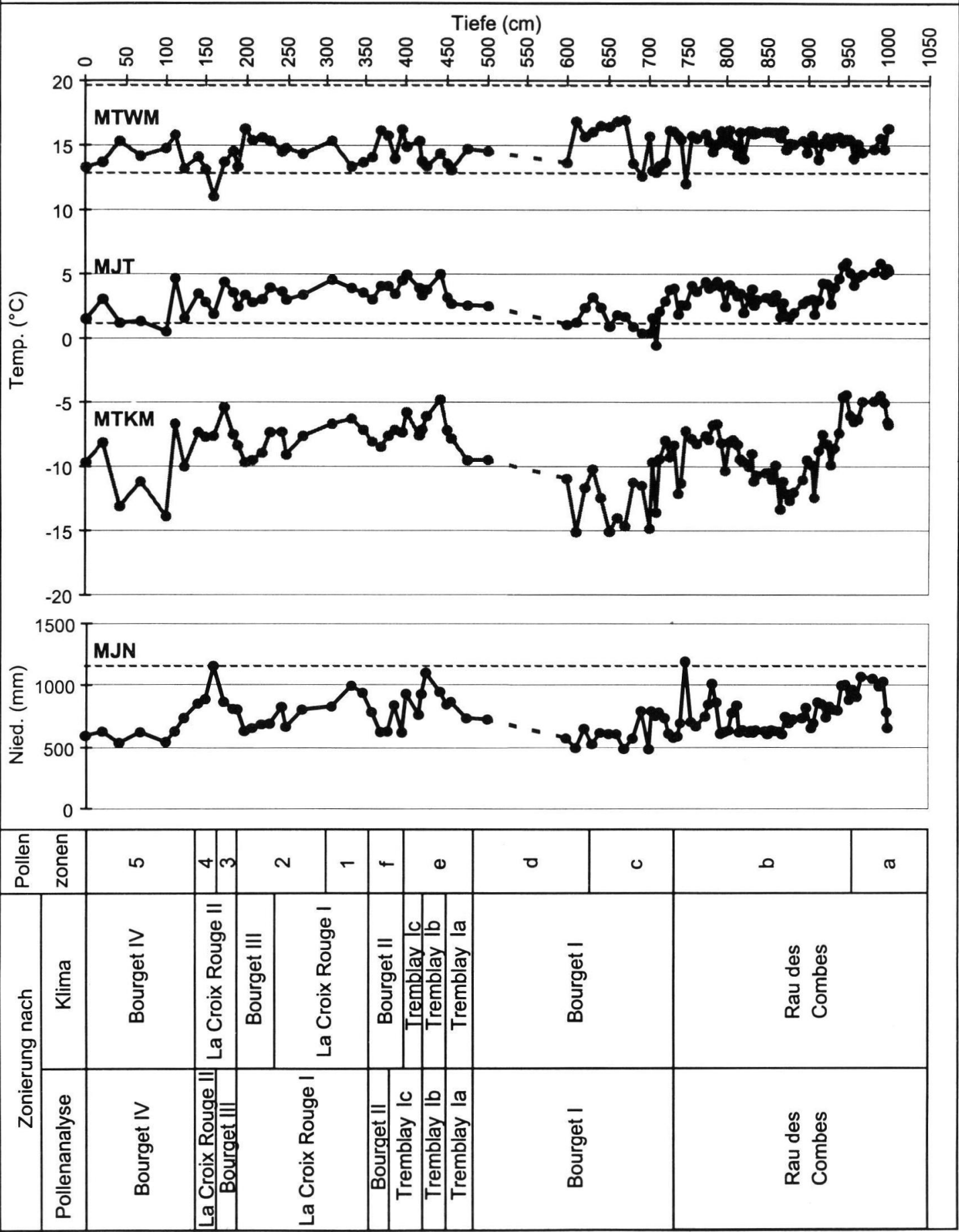

Abb. 8: Klimarekonstruktion mit der Methode der modernen Analoge von Unterhalb La Flachère. Kartographie: S. KLOTZ.

Fig. 8: Climate reconstruction by means of the modern analog vegetation types from "Below La Flachère". Cartography: S. KLOTZ. 
Die Klimarekonstruktion der DA e und f bestätigt im folgenden nicht nur die biostratigraphische Zonierung von Gremmes, der diese Abschnitte dem St. Germain I-Interstadial und dem Melisey II-Stadial zuordnet, sondern zeigt vielmehr auch deutlich deren Untergliederung und klimatische Ausprägung.

Infolge des nahezu gleichzeitigen Einsetzens der Kurven der thermophilen Gehölze, der Zunahme von Picea und dem Rückgang der NBP werden im DA e günstigere Klimaverhältnisse durch den Anstieg der MJT bis auf $5,4^{\circ} \mathrm{C}$ und der MTKM bis auf $-4,5^{\circ} \mathrm{C}$ sowie durch höhere Niederschlagsmengen deutlich. Die wenn auch geringfügige Abnahme der thermophilen Gehölze markiert im weiteren Verlauf einen kurzzeitigen klimatischen Rückschlag, der allerdings anhand der rekonstruierten Werte deutlicher abzulesen ist, und zwar mit einer MJT von nur noch etwa $3,5^{\circ} \mathrm{C}$ und einer um $3^{\circ} \mathrm{C}$ verringerten MTKM. Anschließend werden wieder die Ausgangswerte erreicht, so dass der Verlauf im DA e insgesamt die Folge des St. Germain I-Interstadials mit den Teileinheiten Ia, Ib und Ic nachzeichnet. Ähnliche Rekonstruktionswerte werden im Vergleich dazu für das nördliche Mitteleuropa mit einer MTWM von etwa $15,5^{\circ} \mathrm{C}$ und einer MTKM von $-3^{\circ} \mathrm{C}$ für das St. Germain Ia und Ic sowie eine um $3^{\circ} \mathrm{C}$ geringere MTWM innerhalb des St. Germain Ib genannt (WALKLING, 1997: 87-102). Für Grande Pile ergeben sich allerdings der Methode der modernen Analoge nach um bis zu $4^{\circ} \mathrm{C}$ höhere mittlere Jahrestemperaturen (Guiot et al. 1989: 309-313).

Im DA f macht sich mit einer MJT von $3,2^{\circ} \mathrm{C}$, MTWM um $16,5^{\circ} \mathrm{C}$ und MTKM um $-8^{\circ} \mathrm{C}$ bei zudem geringen Niederschlagsmengen schließlich ein leichter klimatischer Rückgang bemerkbar. Der Abschnitt erscheint im Vergleich zum Melisey IStadial des DA c und d vor allem aufgrund der wesentlich höheren MTKM und MJT weit weniger ausgeprägt. Die höhere Kontinentalität gegenüber dem DA e und die geringeren Anteilswerte thermophiler Gehölze sprechen jedoch für seinen stadialen Charakter, so dass der DA f dem Melisey II-Stadial zugerechnet werden muss. Die Lithostratigraphie wie auch die ein schwach ausgeprägtes Stadial ausweisende Klimarekonstruktion bestätigen, dass zwischen dem St. Germain I und dem nachfolgenden St. Germain II im Grésivaudan kein bedeutender Gletschervorstoß stattgefunden haben kann, wie dies früher angenommen worden ist (HANNSS 1982: 9-60). Wie auch andere Untersuchungen erwiesen haben, ist der Kälterückschlag des Melisey I-Stadial prägnanter entwickelt als der des Melisey II-Stadials (Menke \& TYNnI 1984: 77).

Die Klimarekonstruktion der nachfolgenden DA hat nur noch eine teilweise Übereinstimmung mit den entsprechenden biostratigraphischen Zonierungen ergeben. Vom DA 1 bis Mitte DA 2 ergibt sich infolge des Anstiegs der MJT bis auf $5^{\circ} \mathrm{C}$ bei höherer Ozeanität und der sich anschließenden Abkühlungsphase ein interstadialer Klimaverlauf. Dafür sprechen auch der geringe NBP-Anteil und der höhere Prozentsatz thermophiler Gehölze in diesem DA. Damit und in Übereinstimmung mit der Florenzusammensetzung kann der Abschnitt von DA 1 bis Mitte DA 2 dem St. Germain II zugeordnet werden, das hier im Vergleich zum St. Germain I weniger stark ausgeprägt ist.

Von Mitte DA 2 bis Ende DA 2 folgt nach der Klimarekonstruktion eine stadiale Phase, welche sich aber im Pollenbild nur partiell niederschlägt. In diesem Teilabschnitt wird bei einer MJT um $3^{\circ} \mathrm{C}$, einer MTWM um $16,5^{\circ} \mathrm{C}$ und einer MTKM um $-9,5^{\circ} \mathrm{C}$ eine Abkühlung deutlich, die der im Melisey II-Stadial in DA f ähnlich ist. Diese Phase wird deshalb als Stadial angesehen und infolge der fortlaufenden Bezeichnung dem Ognon I-Stadial zugeschrieben.

Im Anschluss tritt der Wechsel zum DA 3 sehr auffällig hervor, wobei sich dieser DA mit nahezu gleichen klimatischen Verhältnissen wie das St. Germain Ia im DA e präsentiert und der Klimarekonstruktion nach daher dem Ognon I-Interstadial zugeordnet wird. Im Pollenbild weisen die beiden DA jedoch größere Unterschiede auf, zumal im DA 3 die Kiefer über die Fichte dominiert bei zudem geringerer Diversität an thermophilen Gehölzen.

Im Übergang vom DA 3 zu den DA 4 und 5 macht sich trotz variierender MJT-Werte zwischen $0,7^{\circ} \mathrm{C}$ und $5^{\circ} \mathrm{C}$ aufgrund der zunehmenden MTWM und abnehmenden MTKM bei geringer werdenden Niederschlägen auf der Basis der Klimarekonstruktion ein deutlicher klimatischer Rückschlag bemerkbar. Solche klimatischen Verhältnisse findet man heute etwa im SE Finnlands sowie im südlichen Teil Kareliens im NW Russlands. Während das Pollenbild im DA 4 trotz geringen NBP-Anteils, insbesondere von Artemisia, kaum thermophile Gehölze anzeigt und damit eher uneinheitlich erscheint, weist das des DA 5 mit dem Melisey I-Stadial in den DA c und d eine größere Ähnlichkeit auf.

Anhand der Klimarekonstruktion lässt sich insgesamt eindrücklich eine Abfolge warm- und kalt- 
zeitlicher Phasen verfolgen, wobei das "EndEem", Melisey I und Ognon II-Stadial alleine schon durch den Verlauf der MJT markant hervortreten und jeweils im Einklang mit der Zonierung von GREMmeN stehen. Zieht man weiterhin vor allem die MTKM und den MJN in Betracht, so lassen sich nach der klimatischen Zonierung auch das mehrgeteilte St. Germain I, Melisey II, St. Germain II, Ognon II sowie das Ognon I leicht erkennen und voneinander trennen. Gerade der Verlauf der MTKM und des MJN weist darauf hin, dass die warmzeitlichen Verhältnisse hauptsächlich mit höheren Temperaturen des kältesten Monats sowie hohen Niederschlagsmengen einhergehen, die kühlen Phasen dagegen kontinentaleren Charakter besitzen. Hierbei sei allerdings noch einmal angeführt, dass die Klimarekonstruktion Ende DA 2 bis Ende DA 4 nur partiell mit der Zonierung von GREMMEN übereinstimmt, zumal nach der Klimarekonstruktion das Ognon IStadial schon in den Bereich der Pollenzone Ende DA 2 fällt und damit in das endende St. Germain II nach Gremmen. Weiterhin wird für die Pollenzonen DA 3 und 4, die nach Gremmen dem Ognon IStadial und dem Ognon I-Interstadial zugeordet sind, eine geschlossene klimagünstigere Phase rekonstruiert. Sie wird deshalb nach der klimatischen Zonierung ausschließlich dem Ognon I-Interstadial zugerechnet.

Die Rekonstruktion zeigt zudem, dass den warmzeitlichen Abschnitten von Eem, St. Germain I, II und Ognon I jeweils signifikante Klimarückschläge bei noch relativ hohen Niederschlagswerten folgen. Während dabei die Stadiale Melisey II und Ognon I recht gemäßigt erscheinen, sind für das Melisey I und Ognon II-Stadial mit Erniedrigungen der MJT bis zu $10^{\circ} \mathrm{C}$, MTKM bis zu $15,5^{\circ} \mathrm{C}$ und MTKM zwischen $2^{\circ} \mathrm{C}$ und $6^{\circ} \mathrm{C}$ gegenüber den heutigen Verhältnissen Bedingungen vorzufinden, die Gletschervorstöße zumindest in der alpinen Stufe ermöglicht haben können (CHARLESWORTH 1957: 9, 647; FLINT 1971: 66). Insbesondere wird dies bei dem Ognon II-Stadial deutlich, zumal hier die MTWM nur Werte um $14^{\circ} \mathrm{C}$ erreichen. Die Auswirkungen der Abkühlungen im Melisey II und Ognon I-Stadial gemäß der klimatischen Zonierung dürften demzufolge eher als lokal angesehen werden.

\section{Zusammenfassung (Christian HanNSS)}

Zwischen der saalezeitlichen Moräne des OIS 6 und der Weichselmoräne des OIS 4 oder 2 konnten an Moor- und limnischen Sedimenten erstmals inneralpin die drei Hauptwarmphasen des letzten Interglazials 5a, 5c und 5e sensu WOILLARD (1975: 63) mit einer nach oben hin abnehmenden Beteiligung thermophiler Laubbäume nachgewiesen werden. Nach der Definition von GrEMMEN und WoIllard sind dies das Rau des CombesInterglazial $(=$ Eem $)$ sowie das Tremblay- $(=$ St. Germain I) und das La Croix Rouge I- (= St. Germain II) Interstadial. Im Unterschied zum Val du Bourget gelang es in Unterhalb La Flachère, die palynostratigraphischen Äquivalente der OIS 5a, $5 \mathrm{c}$ und $5 \mathrm{e}$ komplett in einer einzigen Abfolge pollenanalytisch zu erfassen. Im Val du Bourget sind diese drei Warmphasen palynologisch bisher nur lithostratigraphisch getrennt in einer aus dem Eem und St. Germain I bestehenden unteren Sequenz nachgewiesen und einer zweiten oberen mit dem St. Germain II und vier darüber entwickelten frühestweichselzeitlichen Kiefern-Fichten-Interstadialen.

Eine lithostratigraphisch durchlaufende letztinterglazial- bis frühestweichselzeitliche Abfolge wurde pollenanalytisch in den französischen Nordalpen noch in den Banquetten im SW des Lac d'Annecy von A. Brun (unveröffentlicht) erarbeitet. Eine zweite liegt aller Wahrscheinlichkeit nach am Rau de l'Amourette im südlich von Grenoble gelegenen Trièves vor. Dort konnte jedoch das OIS $5 \mathrm{a}$ (= St. Germain II) bisher nur malakologisch und nicht wie die OIS $5 \mathrm{c}$ und $5 \mathrm{e}$ auch palynologisch dokumentiert werden (GREmmen et al. 1984: 93, 96). Hinsichtlich Temperatur und Niederschlag liefert die Klimarekonstruktion zur letztinterglazialzeitlichen Abfolge von Unterhalb La Flachère ein deutliches Bild, welches das in Schüben kühler werdende endende Eem im Übergang zu den frühestweichselzeitlichen Kiefern-Fichten-Interstadialen zeigt. Aufgrund absoluter Temperaturwerte und dem jeweiligen Grad an Kontinentalität können die einzelnen durch Rekonstruktion bestimmten klimatischen Zonen einfach voneinander unterschieden und zum größten Teil auch mit den biostratigraphischen Ergebnissen in Einklang gebracht werden.

\section{Dank}

Die Autoren danken der DFG für die Finanzierung der Kernbohrung von Unterhalb La Flachère sowie den beiden Bohrmeistern BERNARD und BANET der Firma Bachy aus Meyzieu für all die Anstrengungen, die nötig waren, um die Bohrkerne in ihrer außergewöhnlichen Qualität zu gewinnen. Dank gebührt auch Herrn Prof. Dr. Christian SCHLÜCHTER und einem anonymen Gutachter für die kritische Durchsicht des Manuskriptes. 


\section{Literatur}

Bartlein, P. J., Prentice, I. C. \& Webb, T. III. (1986): Climatic response surfaces from pollen data for some eastern North American taxa. - Journal of Biogeography, 13: 35-57, Oxford.

Beaulieu, J.-L. De, Eicher, u. \& Monjuvent, G. (1994): Reconstruction of Middle Pleistocene palaeoenvironments based on pollen and stable isotope investigations at Val-de-Lans, Isère, France. - Vegetation History and Archaeobotany, 3: 127-142; Berlin.

- \& Reille, M. (1984): A long Upper Pleistocene pollen record from Les Echets, near Lyon, France. - Boreas 13: 111-132, Oslo.

Bourdier, F. (1961-62): Le bassin du Rhône au Quaternaire, 2 Bde. - Editions du centre national de la recherche scientifique: 364 S.; Paris.

Brun, A. \& Hannss, Ch. (1998): La fiabilité des datations au ${ }^{14} \mathrm{C}$ pour des séquences fini-pléistocènes et les différentes phases de la glaciation wurmienne: l'exemple des Alpes Orientales et de leur avant-pays. Revue de Géographie Alpine, 86: 9-25, 91.

Caspers, G. \& Freund, H. (1997): Die Vegetations- und Klimaentwicklung des Weichsel-Früh- und -Hochglazials im nördlichen Mitteleuropa. - Schriftenreihe der dt. geol. Ges., 4: 201-249; Hannover.

Charlesworth, J. K. (1957): The Quaternary Era, 1: 591 p., 2: 1700 p.; London.

Depape, G. \& Bourdier, F. (1952a): La flore interglaciaire à Rhododendron ponticum L. de Barraux dans la vallée de l'Isère, entre Grenoble et Chambéry. Comptes Rendus Hebdomadaires des Séances de l'Académie des Sciences, 235: 1531-1533; Paris.

- (1952b): Le gisement interglaciare à Rhododendron ponticum L. de Barraux dans la vallée de l'Isère, entre Grenoble et Chambéry. - Travaux du Laboratoire de Géologie de la Faculté des Sciences de l'Université de Grenoble, 30: 81-102; Grenoble.

Eissmann, L. (1997): Das quartäre Eiszeitalter in Sachsen und Nordostthüringen. - Altenburger naturwiss. Forschungen, 8: 98 S.; Altenburg.

FurNT, R. F. (1971): Glacial and Quaternary Geology.892 p.; New York (John Wiley).

Frevzel, B. (1968): Grundzüge der pleistozänen Vegetationsgeschichte Nord-Eurasiens. - 326 S.; Wiesbaden (Steiner).

- (1991): Klimageschichtliche Probleme der letzten 130000 Jahre. - 451 S.; Stuttgart (Fischer).

Gras, S. (1851): Banc de lignite observé à Barraux. - Bulletin de la Société Scientifique de l'Isère, 11, série 2: 198; Rennes.

Gremmen, W. H. E. (1982): Palynological investigations of Late-Pleistocene deposits in southeastern France. - 94 p.; Groningen (Selbstverlag).

\& Hannss, CH. (1994): Une nouvelle analyse pollinique de la banquette du Tremblay et son rapport avec le Pléistocène supérieur du Val du Bourget (Savoie). - Géologie Alpine, 70: 29-45; Grenoble.

- Hannss, Ch. \& Puissegur, J. (1984): Die warmzeitlichen Ablagerungen am Rau de l'Amourette (Trièves, französische Alpen). - Eiszeitalter und Gegenwart, 34: 87-103; Stuttgart.

Grichuk, V. P. (1969): An attempt to reconstruct certain elements of the climate of the northern hemisphere in the Atlantic period of the Holocene. - Golotsen, Akad. Nauk SSSR, Inst. Geogr., Moscow Nauka: 4157; Moskau.

- Gurtovaya, Y., Zelikson, E. M. \& Borisova, O. K. (1984): Methods and results of Late Pleistocene paleoclimatic reconstructions. - Late Quaternary environments of the Soviet Union: 251-260; Minneapolis.

Guiot, J. (1987): Late Quaternary climatic change in France estimated from multivariate pollen time series. - Quaternary Research, 28: 100-118; New York.

- (1990): Methodology of the last climatic cycle reconstruction in France from pollen data. - Paleogeography, Paleoclimatology, Paleoecology, 80: 49-69; Amsterdam.

- Beaulieu, J. L. De, Cheddadi, R., David, F., Ponel, P. \& Rellle, M. (1993): The climate in Western Europe during the last Glacial/Interglacial cycle derived from pollen and insect remains. - Palaeogeography, Palaeoclimatology, Palaeoecology, 103: 73-93; Amsterdam.

- Pons, A., Beaulieu, J. L. De \& Reille, M. (1989): A 140000 -year continental climate reconstruction from two European pollen records. - Nature, 338: 309-313; London.

Hannss, Ch. (1974): Premières indications par une datation au ${ }^{14} \mathrm{C}$ pour l'âge interstadiaire würmien des dépôts fluviatiles de la banquette de Barraux (rive droite du Haut-Grésivaudan). - Revue de Géographie Alpine, 62: 551-554; Grenoble.

- (1982): Spätpleistozäne bis postglaziale Talverschüttungs- und Vergletscherungsphasen im Bereich des Sillon alpin der französischen Nordalpen.

Mitteilungen der Kommission für Quartärforschung der Österreichischen Akademie der Wissenschaften, 4: 213 S.; Wien.

- (1983): Neuere Ergebnisse über den Ablauf des Spätpleistozäns im Bereich des zentralen Sillon alpin in den französischen Nordalpen. - In: SCHRÖDERLanz, H. [Hrsg.]: Kolloquium, Trier, 1980. 05. 15-17. Spät- und postglaziale Gletscherschwankungen: Glazial- und Periglazialformen: 15-33; Rotterdam.

- Bottema, S., Grootes, P.-M., Koster, Y. M. \& MünNICH, M. (1976): Nouveaux résultats sur la stratigraphie et l'âge de la banquette de Barraux (Haut-Grésivaudan, Isère). - Revue de Géographie Alpine, 64 : 495-513; Grenoble.

Howe, S. \& WeBB, T. III. (1983): Calibrating pollen data in climatic terms. Improving the methods. - Quaternary Science Reviews, 2: 17-51; Oxford.

Huntley, B. (1992): Pollen-climate response surface and the study of climate change. - Quaternary Proceedings, 2: 91-99; Cambridge.

Imbrie, J. \& Kipp, N. G. (1971): A new micropaleontological method for quantitative paleoclimatology; application to a late Pleistocene Caribbean core. - In: The late Cenozoic glacial ages: 71-147; New HavenLondon.

Iversen, J. (1944): Viscum, Hedera and Ilex as climate indicators. A contribution to the study of the post-glacial temperature climate .- Geologiska Foereningen i Stockholm Foerhandlingar, 66: 463-483; Stockholm. 
KrbetscheK, M. R. \& Stolz, W. (1994): Lumineszenz-Datierungen an pleistozänen Sedimenten aus Tagebauen des mitteldeutschen und Lausitzer Braunkohlenreviers. - Altenburger naturwiss. Forschungen, 7: 289-295; Altenburg.

LIEDTKE, H. (1981): Die nordischen Vereisungen in Mitteleuropa. - Zentralausschuss für dt. Landeskunde, 204: 307 S.; Trier.

Menkf, B. \& Trnnt, R. (1984): Das Eeminterglazial und das Weichselfrühglazial von Redderstall/Dithmarschen und ihre Bedeutung für die mitteleuropäische Jungpleistozän- Gliederung .- Geol. Jb. A76: $117 \mathrm{~S}$; Stuttgart.

Monjuvent, G. (1969): Nouvelles datations absolues dans les formations quaternaires du Sillon alpin. Revue de Géographie Alpine, 57: 545-558; Grenoble.

Mosbrugger, V. \& Utescher, T. (1997): The coexistence approach - a method for quantitative reconstructions of Tertiary terrestrial palaeoclimate data using plant fossils. - Palaeogeography, Palaeoclimatology, Palaeoecology, 134: 61-86; Amsterdam.

Müller, M. J. (1980): Handbuch ausgewählter Klimastationen der Erde. - Forschungsstelle Bodenerosion der Universität Trier: 346 S.; Trier.

Peyron, O., Gutot, J., Cheddadi, R., Tarasov, P., Reille, M., Beallieu, J. L. ie, Bottema, S. \& Andrieu, V. (1998): Climatic reconstruction in Europe for 18000 yr B.P. from pollen data. - Quaternary Research, 49: 183-196; New York.

Reille, M., Andrieu, V., Beatlifu, J.-L. De, Guenet, P., Goeurey, C. (1998): A long pollen record from Lac du Bouchet, Massif central: for the period ca. 325 to $100 \mathrm{ka} \mathrm{BP}$ (= OIS 9c to OIS 5e). - Quaternary Science Reviews, 17: 1107-1123; New York.

Schlïchter, CH. (1976): Geologische Untersuchungen im Quartär des Aaretals südlich von Bern (Stratigraphie, Sedimentologie, Paläontologie). - Beiträge zur Geologischen Karte der Schweiz, Neue Folge, 148; Bern.
Urban, B. (1996): Mittelpleistozäne Waldzeiten im Tagebau Schöningen: Spektren aus dem Holstein-Interglazial und dem Harbke-Interstadial. - Tübinger Monographien zur Urgeschichte, 11: 487-495; Tübingen.

WALKLING, A. (1997): Käferfundliche Untersuchungen an weichselzeitlichen Ablagerungen der Bohrung Grols Todtshorn (Kr. Harburg, Niedersachsen). Schriftenreihe der dt. geol. Ges., 4: 87-102; Hannover.

- \& COOpE, G. R. (1996): Climatic reconstructions from the Eemian/Early Weichselian transition in central Europe based on the coleopteran record from Gröbern, Germany. - Boreas, 25: 145-159; Oslo.

WegmülLER, S. (1992): Vegetationsgeschichtliche und stratigraphische Untersuchungen an Schieferkohlen des nördlichen Alpenvorlandes. - Denkschrift der Schweizerischen Akademie der Naturwissenschaften, 102: $82 \mathrm{~S}$; Basel.

Welten, M. (1982): Pollenanalytische Untersuchungen im Jüngeren Quartär des nördlichen Alpenvorlandes der Schweiz. Textband. - Beiträge zur Geologischen Karte der Schweiz, N.F. 156; Bern.

WollLard, G. (1975): Recherches palynologiques sur le Pléistocène dans l'Est de la Belgique et dans les Vosges Lorraines. - Acta Geographica Lovaniensia, 14: 118 p.; Louvain.

- (1978): Grande Pile Peat Bog: A continous Pollen Record for the Last 140000 Years. - Quaternary Research, 9: 1-21; New York.

Manuskript eingegangen am 18. 01. 1999, zum Druck angenommen am 03. 07. 2000 